GRÉGORY BRESSOLLES

Chaire Business in a Connected World

KEDGE Business School - Bordeaux

CATHERINE VIOT

Laboratoire SAF (Sciences Actuarielle et Financière) - Université Lyon 1

Systèmes d'Information et Management

Pour citer cet article :

Bressolles Grégory, Viot Catherine, «L'intégration des canaux de distribution en contexte de transition digitale : une relecture par la théorie des ressources », Systèmes d'information \& management, 2021/1 (Volume 26), p. 9-44.

URL : https://www.cairn.info/revue-systemes-d-information-et-management-2021-1-page-

$\underline{9 . h t m}$

\title{
L'intégration des canaux de distribution en contexte de transition digitale : une relecture par la théorie des ressources
}

\section{Résumé}

De nombreux distributeurs s'engagent dans une stratégie d'intégration des canaux sous la pression du consommateur qui souhaite désormais une expérience d'achat intégrée. L'intégration des canaux s'inscrit dans le contexte de la transition digitale de l'entreprise et afin de l'aborder, il est nécessaire de se situer à un niveau stratégique. En effet, l'intégration des canaux s'accompagne nécessairement d'une collaboration inter-fonctionnelle. En se basant sur le cadre de la théorie des ressources (RBV), cet article se propose de répondre à la question de recherche suivante : quelles sont les ressources mobilisées par les distributeurs en vue d'une intégration réussie ? Ainsi, l'analyse des ressources mobilisées par les distributeurs est réalisée à partir de la classification des ressources logistiques de Duong et Paché (2015) qui est utilisée comme grille de lecture après adaptation au contexte de l'intégration des canaux.

Une étude qualitative exploratoire reposant sur six cas met au jour les ressources déployées dans leurs stratégies d'intégration par des distributeurs français et canadiens dans trois secteurs. Les résultats montrent que les distributeurs mobilisent cinq ressources: des ressources technologiques, organisationnelles, physiques, relationnelles et d'expertise. Cette recherche confronte, pour la première fois, la grille de Duong et Paché (2015) à un terrain empirique et élargit son application au-delà de son contexte d'origine, la logistique. D'un point de vue théorique, cet article fait émerger et structure une réflexion autour des ressources nécessaires au déploiement d'une stratégie omnicanal. D'un point de vue managérial, il souligne la diversité des ressources qui sous-tend les stratégies d'intégration des canaux et fournit aux équipes dirigeantes un outil permettant d'établir un diagnostic des ressources.

\section{Mots clés}

Omnicanal; Théorie des ressources; Intégration; Systèmes d'information; Logistique ; Technologies de l'Information (T.I.) 
Remerciements : les auteurs tiennent à remercier la rédactrice en chef adjointe ainsi que les deux évaluateurs anonymes pour la pertinence de leurs remarques et suggestions qui ont permis d'améliorer significativement les contributions de cet article. 


\title{
Integration of distribution channels in the context of digital transition: A Resource-Based View perspective
}

\begin{abstract}
Channels' integration is one of the strategies that retailers have to pursue in order to deliver a "seamless" consumer shopping experience. Channels' integration is part of the digital transition of a company and, in order to study this transition, a strategic-level perspective is necessary. Indeed, channels' integration involves inter-functional collaboration. Grounded in the Resource-Based View theory (RBV), this article aims to answer the following research question: what resources retailers have to leverage for successful channels integration? Thus, the analysis of resources leveraged by retailers is carried out according to the Duong and Paché's (2015) classification of logistical resources which is used as a reading grid adapted to the context of channels integration.

An exploratory qualitative study based on six cases sheds light on the resources implemented by French and Canadian retailers in three sectors in their integration strategies. The findings show that retailers implement five resources: technological, organizational, physical, relational and expertise's resources. In addition, this study is the first to confront the Duong and Paché's (2015) grid with an empirical field and extends its application beyond the initial context of logistics. From a theoretical point of view, this article brings out and structures a reflection around the required resources for implementing an omnichannel strategy. From a managerial point of view, this research highlights the diversity of resources that underpin channel integration strategies and provides a tool for managers in order to establish diagnosis of resources.
\end{abstract}

\section{Keywords}

Ominichannel; Resource Based View; Integration; Information Systems; Logistics; Information Technologies (I.T.) 


\section{Introduction}

En 2019, JouéClub, leader de la vente de jouets en France, a opéré sa transition digitale vers le commerce omnicanal. Interviewée par la revue LSA ${ }^{1}$, Aurélie Cayla, la responsable marketing digital et e-commerce de JouéClub, indiquait: "Auparavant, nous disposions de deux plateformes, une dédiée au e-commerce avec livraison à domicile et une autre, JouéClub Drive, destinée au click-\&-collect, renvoyant vers les 221 magasins de nos adhérents ». Cette organisation a été abandonnée car elle ne favorisait pas la fluidité du parcours client. La solution mise en place par JouéClub a permis de regrouper sur une seule plateforme l'ensemble de son offre et de ses services. La vision de l'omnicanal chez JouéClub est la suivante : «Un réseau, une enseigne et un client à satisfaire, peu importe le moyen par lequel il arrive chez nous. Désormais, sur notre site, le client peut choisir de se faire livrer ou de venir récupérer son achat dans le magasin de son choix, en fonction de la disponibilité des produits. » Si cette nouvelle organisation apporte des réponses à la problématique du parcours client, elle a nécessité de nombreux changements en interne, notamment du point de vue de l'intégration des systèmes d'information (SI). Le nouveau SI se traduit par la synchronisation en temps réel des stocks de tous les magasins; il s'est accompagné de la refonte du système de management des fiches produits et de la fusion des profils clients.

Comme l'illustre l'exemple de JouéClub, après la mise en place du multicanal, le commerce connait une nouvelle phase de changement dont la raison sous-jacente est l'intégration des canaux. Comme le soulignaient Paché et al. (2014), le fait qu'encore trop peu d'entreprises travaillent sur des schémas globalisants en matière d'intégration des canaux peut déboucher sur des dysfonctionnements : des risques de conflits et un manque de synergies potentielles entre canaux, ainsi qu'une période d'apprentissage longue et coûteuse pour l'entreprise (Paché et al., 2014), synonyme de perte potentielle d'avantage concurrentiel (Vanhemms, 2009). Ce changement résulte des effets combinés des innovations dans le domaine des technologies de l'information (TI), ainsi que des nouveaux comportements adoptés par les consommateurs. Ces derniers utilisent, en effet, les smartphones, tablettes, etc. dans de nombreux parcours d'achat (Rigby, 2011). Ce consommateur connecté, parfois décrit comme «channel agnostic » (Aubrey et Judge, 2012), utilise les canaux, de manière interchangeable, durant le processus de recherche et d'achat (Verhoef et al., 2015).

Les attentes de ce consommateur "augmenté » ont évolué : il souhaite désormais une expérience d'achat intégrée entre les différents canaux (Aubrey et Judge, 2012 ; Piotrowicz et Cuthbertson, 2014). Les distributeurs sont donc contraints de s'adapter à ces nouvelles attentes des clients de peur de voir le rôle du magasin évoluer vers celui de simple showroom où les consommateurs viendraient rechercher de l'information, avant l'achat, et/ou vers celui de point de retrait/retour des marchandises commandées par le canal digital, qu'il s'agisse d'un achat via un ordinateur, un smartphone, une tablette ou même une enceinte connectée.

Une des réponses envisagées par les distributeurs est de s'engager dans une stratégie d'intégration des canaux afin d'offrir aux consommateurs, une expérience d'achat «sans couture ». L'intégration des canaux fait référence au degré auquel une firme coordonne les objectifs, le design et le déploiement de ses canaux afin de créer des synergies pour elle-même et d'offrir des bénéfices particuliers à ses clients (Cao et Li, 2015). L'intégration des canaux serait imposée par les évolutions du marché (clients et concurrents).

L'expérience d'achat sans couture offerte au client ne constitue que la partie visible de l'intégration des canaux. Pour le distributeur, cette intégration s'appuie sur des ressources peu visibles pour le consommateur. Parmi celles-ci, figurent un ensemble de ressources comme un

${ }^{1}$ LSA, https://www.lsa-conso.fr/comment-joueclub-a-fait-sa-mue-digitale, 319972 
SI adapté (Luo et al., 2016), une supply chain et une gestion logistique rendant possible le commerce omnicanal (Jeanpert et Paché, 2016; Fabbe-Costes et Jahre, 2008) et des compétences spécifiques des ressources humaines (RH) (Oh et al., 2012).

Exacerbées avec le déploiement de l'omnicanal, les problématiques d'intégration étaient déjà évoquées dans la littérature à propos du multicanal. La distribution multicanal poussait les distributeurs à s'interroger sur une intégration fonctionnelle dans le domaine du marketing, des inventaires, du traitement des commandes et des retours produits pour que les opérations logistiques soient rationalisées (Mollenkopf et al., 2007). Avec l'évolution vers l'omnicanal, «la dimension électronique s'intègre dans l'ensemble des canaux y compris dans les magasins » (Isaac, 2017 : p. 41). Il est alors nécessaire de recourir de manière plus systématique aux TI afin d'intégrer les ressources et les opérations du canal physique et digital (Oh et al., 2012).

Si la littérature sur le multicanal abonde, celle concernant l'omnicanal est plus rare (Saghiri et al., 2017). Ces auteurs soulignent de nombreuses contradictions dans la littérature relative au multicanal justifiant de nouvelles recherches centrées sur l'omnicanal. De plus, Luo et al. (2016) soulignent à propos de l'omnicanal : "little research has investigated the underlying mechanism of how IT combines with other organizational resources change business processes and help firms cope with intensified competition" (p. 309). Cette recherche répond à ces appels en s'interrogeant sur les mécanismes d'intégration des ressources qui sous-tendent l'omnicanal. La question de recherche peut être formulée ainsi : quelles sont les ressources mobilisées par les distributeurs en vue de l'intégration des canaux ?

Cette analyse des ressources sera effectuée sous l'angle des SI, de la logistique \& de la supply chain et des RH. En effet, l'intégration des canaux représente pour les distributeurs des investissements coûteux occasionnés par l'intégration des SI existants (ou le développement d'un nouveau SI) ainsi que l'intégration de la logistique \& de la supply chain ayant pour corolaire l'adaptation ou la création de nouveaux entrepôts permettant l'intégration des assortiments. A ces investissements s'ajoute l'accompagnement du personnel pour le préparer au changement et réduire les résistances : formation des vendeurs aux technologies digitales en magasin (TDM), aux nouveaux processus ou aux parcours d'achat intégrés, etc.

De tels investissements ne peuvent être acceptés par les distributeurs que s'ils en espèrent un retour qui pourrait prendre la forme d'un avantage concurrentiel. Ce raisonnement conduit certains auteurs à considérer que l'intégration des canaux s'appuie sur des ressources pouvant mener à l'acquisition d'un tel avantage (par exemple, Oh et al., 2012). Ces derniers étudient les effets de complémentarité de l'utilisation des TI et des RH dans le cas de l'intégration des canaux de distribution. Le cadre théorique qu'ils évoquent est celui de la resource-based view - RBV (Wernerfelt, 1984). Récemment, Duong et Paché (2015) ont complété le cadre de la théorie des ressources (RBV) en proposant une classification des ressources adaptée au contexte de la logistique en cinq catégories : ressources technologiques, physiques, organisationnelles, relationnelles et d'expertise. Les ressources technologiques font référence, dans cette catégorisation, au système d'information (SI) dans une acception large (matériel, logiciels, données, technologies liées à Internet, etc.). Les SI, et plus largement les TI, sont au cœur de l'intégration des canaux (Luo et al., 2016): "For the retail industry, technology is breaking down the barriers between different retail channels and is making omnichannel retailing inevitable - an integrated sales experience that melds touch-and-feel information in the physical world with online content".

De nombreuses recherches centrées sur l'intégration des canaux de distribution ne font pas référence à un cadre théorique spécifique (ce qui est, par exemple, le cas des recherches récemment menées par Adivar et al., 2019, Aktur et al., 2018, Larke et al., 2018). Lorsque les 
recherches y font expressément référence, il ne semble pas y avoir de consensus sur celui-ci : théorie de l'avantage concurrentiel retenue par Ishfaq et al. (2016), celle des systèmes adaptatifs complexes par Saghiri et al. (2017) ou encore théorie des ressources (RBV) mobilisée par Oh et al. (2012) ainsi que par Luo et al. (2016).

Les deux articles qui s'intéressent à la combinaison des TI avec d'autres ressources organisationnelles dans le contexte des stratégies d'intégration des canaux (Oh et al., 2012 ; Luo et al., 2016) ont validé la pertinence du cadre théorique de la RBV. Notre recherche se situe dans ce même courant et retient donc la RBV comme cadre théorique. Pour cela, nous mobilisons des travaux issus de différents champs du management concernés par l'intégration des canaux : les TI, les RH, ainsi que des travaux relevant de la logistique (Duong et Paché, 2015) et notamment la grille de lecture que proposent ces auteurs pour analyser les ressources nécessaires à l'acquisition d'un avantage concurrentiel dans les activités de logistique. Si certains auteurs ont souligné l'intérêt de combiner les ressources relevant des TI avec d'autres ressources organisationnelles, notamment les RH (Oh et al., 2012 ; Luo et al., 2016), il n'existe pas, à notre connaissance, de travaux intégrant simultanément les SI, les RH et la logistique.

Ainsi, cet article se propose d'examiner les ressources nécessaires pour une intégration réussie des canaux de distribution. L'objectif de cet article est de faire émerger et de structurer une réflexion autour des ressources nécessaires au déploiement d'une stratégie omnicanal. Cette réflexion est fondée sur le cadre théorique de la RBV et est structurée selon la classification des ressources logistiques de Duong et Paché (2015) qui est, dans le présent article, utilisée comme grille de lecture et adaptée à l'intégration des canaux. Cette recherche de nature exploratoire est, de plus, l'occasion de confronter à un terrain empirique la grille de Duong et Paché (2015) qui, à notre connaissance, n'a pas fait l'objet d'une telle application systématique, ni n'a été élargie au-delà de son contexte d'origine.

Les nouvelles technologies telles qu'Internet et les technologies digitales en magasin ont modifié le champ concurrentiel du commerce de détail (Luo et al., 2016). Dans ce nouveau contexte concurrentiel, l'intégration est considérée comme une priorité par les distributeurs car elle conditionne leur survie et leur compétitivité (Adivar et al., 2019, p. 257). Ils ont donc besoin d'une meilleure compréhension des déterminants de ce succès (Adivar et al., 2019, p. 258). D'un point de vue managérial, cette recherche permet d'aider les distributeurs dans leur processus de transformation digitale en leur proposant un outil de diagnostic des ressources et des recommandations adaptées aux cinq catégories de ressources. Cependant, la nature exploratoire de l'étude empirique, limite la généralisation des résultats.

La revue de littérature (1) rappelle les mécanismes d'intégration sous-jacents à l'œuvre dans les stratégies omnicanal, puis souligne les facteurs clés de succès de cette intégration. La pertinence du cadre théorique de la RBV assorti de la grille de Duong et Paché (2015) est argumentée. Cette grille est ensuite adaptée puis utilisée dans l'étude qualitative exploratoire (2). Les résultats de l'étude menée auprès de six distributeurs français et canadiens sont ensuite présentés selon les cinq ressources proposées par Duong et Paché (2015) (3), puis discutés (4).

\section{Revue de littérature}

La mise en œuvre de stratégies d'intégration des canaux par les distributeurs est relativement récente, de même que la littérature qui lui est consacrée (Saghiri et al., 2017, p. 54 ; Cao et Li, 2018 , p. 1). Elle se traduit par une évolution des termes employés dans la profession, du multicanal à l'omnicanal. L'omnicanal est la forme la plus aboutie de cette intégration. 


\subsection{L'intégration : le fondement d'une stratégie omnicanal}

On ne peut aborder la question de l'intégration des canaux de distribution sans évoquer la manière dont le système de distribution d'une enseigne est organisé. Les termes «multi- » et « omnicanal » correspondent à des situations spécifiques qui ont fait l'objet de clarifications par Beck et Rygl (2015 ; p. 174). Le multicanal se distingue de l'omnicanal :

- Multicanal "selling merchandise or services through more than one channel or all widespread channels, whereby the customer cannot trigger channel interaction and/or the retailer does not control channel integration”

- Omnicanal "selling merchandise or services through all widespread channels, whereby the customer can trigger full channel interaction and/or the retailer controls full channel integration".

Évoluer d'une stratégie multicanal vers une stratégie omnicanal est synonyme d'abandon d'une organisation en silos (Gallino et Moreno, 2014 ; Piotrowicz et al., 2014) au profit d'une intégration des canaux (Cao, 2014). Dans l'organisation en silos, les canaux sont traités séparément et sont souvent gérés par des départements distincts qui ne coopèrent pas toujours pleinement, ce qui a pour conséquence une faible intégration en matière de prix, de promotions, de stratégie de marque, de gestion de la logistique \& de la supply chain et d'expérience délivrée au client (Piotrowicz et al., 2014).

Lors de l'apparition du e-commerce, les distributeurs se sont d'abord tournés vers une organisation en silos, la considérant comme préférable, car elle permettait de cibler les clients en fonction de leur canal de prédilection et réduisait les risques de cannibalisation (Cao, 2014). Cette position pouvait se justifier lorsque le commerce électronique en était à ses balbutiements et que les acheteurs en ligne étaient rares et présentaient un profil typé. L'achat en ligne s'est ensuite démocratisé et les arguments qui avaient prévalu au choix d'une organisation en silos ont perdu de leur intérêt, d'autant plus que les consommateurs ne percevaient pas les frontières entre les canaux et surfaient de l'un à l'autre, pour un même achat. Plusieurs recherches ont ensuite démontré empiriquement que les risques de cannibalisation entre canaux avaient été surestimés (Deleersnyder et al., 2002 ; Xu et al., 2014). L'idée d'une intégration des canaux, définie comme "the degree to which different channels interact with each other" (Bendoly et al., 2005) ou comme "the extent to which channels share common organizational resources, including departments such as marketing, finance, and logistics" (Tate et al., 2005), s'est peu à peu imposée.

Des études récentes montrent que l'intégration des canaux est source de performance pour la firme en termes de vente ( $\mathrm{Cao}$ et $\mathrm{Li}, 2015)$ et d'avantage concurrentiel pour le distributeur (Herhausen et al., 2015). Elle peut enrichir la proposition de valeur faite au client (Gallino et Moreno, 2014) et accroître la propension au « research shopping » (propension à faire une recherche sur un canal et à acheter par le biais d'un autre canal). Enfin, le déploiement de politiques marketing intégrées entre canaux en matière de communication, de prix et d'assortiment, a un effet positif sur les ventes parce que la rétention entre canaux augmente (Van Baal, 2014). Cependant, l'intégration des canaux peut conduire à un jeu à somme nulle lorsque l'avantage d'un canal est effacé par les désavantages d'un autre canal ce qui accroît le risque de di-synergies ou de cannibalisation (Falk et al., 2007).

\subsection{Les facteurs clés d'une intégration réussie des canaux de distribution}

L'intégration des canaux de distribution est une décision stratégique dont le succès repose sur un ensemble de ressources qui doivent être maîtrisées en interne. La littérature, bien que peu nombreuse en ce qui concerne l'omnicanal, identifie un certain nombre de facteurs facilitant l'intégration des canaux (Hübner et al., 2016 ; Lewis et al., 2014 ; Oh et al., 2012 ; Spreer et 
Rauschnabel, 2016). Parmi ces facteurs, l'intégration des SI occupe une place centrale. L'intégration repose également sur des ressources liées à la logistique \& la supply chain (la gestion intégrée des stocks et des assortiments, la coordination des opérations entre les canaux, etc.). Il n'est pas surprenant d'évoquer ces ressources comme piliers de l'intégration des canaux. En effet, Fabbe-Costes (2002a) soulignait les liens étroits entre supply chain et SI. Enfin, l'intégration des canaux doit s'appuyer sur les RH.

\subsubsection{L'intégration des Systèmes d'Information}

La question de l'intégration des SI est un thème classique et d'actualité autant pour les praticiens que pour les chercheurs (Bidan et al., 2012 ; de Corbière, Rowe et Wolff, 2012 ; Gorkhali et Xu, 2019; Yin et al., 2019). L'intégration des SI est présentée comme une brique essentielle de l'intégration des entreprises (Tchokogué et al., 2008). Comme le soulignent Chalmeta et al. (2001, p. 176) « un objectif fondamental sous-jacent à tout projet d'intégration de l'entreprise est la création d'une infrastructure globale de l'information à partir des TI; cette structure devant être flexible et efficace ». A propos de l'intégration des SI, Fabbe-Costes (2002a, p. 81) évoque les difficultés liées à l'alignement de SI autonomes (intra- et interorganisationnels) devant échanger ou partager des informations, voire inter-fonctionner. Cette difficulté concerne l'intégration des canaux de distribution lorsque chaque canal repose sur un SI quasi autonome. Il est alors nécessaire de construire un SI qui permet d'intégrer les données à travers les différents canaux et de les analyser de manière holistique (Lewis et al, 2014).

Reix et al. (2016) définissent un SI comme un ensemble organisé de ressources : matériel, logiciel, personnel, données, procédures permettant d'acquérir, de traiter, de stocker, de communiquer des informations (sous forme de données, textes, sons, images, etc.) dans et entre des organisations. Dans le contexte de l'intégration des canaux, le matériel fait référence à la technologie digitale qui peut être celle des clients (smartphone), celle qui équipe les vendeurs (tablette, smartphone, montre connectée, etc.) ainsi que la technologie en magasin (écrans tactiles, bornes, etc.). Les données sont celles liées au chiffre d'affaires, aux parcours clients, aux inventaires de stocks en temps réel, etc. Le personnel fait référence aux conseillers/vendeurs en contact avec le client, mais aussi l'encadrement et les dirigeants. Enfin, des procédures spécifiques, comme la création d'un compte Internet pour le client en magasin, l'enregistrement de ventes additionnelles, suite à un retrait en magasin d'une commande passée en ligne, sont souvent associées à l'intégration des canaux.

La notion d'intégration des SI renvoie au processus de mise en relation des différentes parties du SI pour un partage d'informations utiles (Bidan, 2004). Seddon et al. (2010) définissent l'intégration des SI comme «l'unification des processus, systèmes et/ou données depuis de multiples systèmes informatisés et ce, pas nécessairement dans une seule organisation », d'où l'idée d'une intégration interne et externe des SI. Le point de vue adopté dans la présente recherche est davantage celui de l'intégration interne des SI qui, selon Hammer (2001), fait référence à " a firm's electronic links in information technology applications to data acquisition and storage systems that facilitate the sharing of accurate and timely information in support of cross-functional processes. » En effet, l'angle d'approche retenu est celui du distributeur confronté à l'intégration de canaux de distribution jusque-là organisés en silos. Bien sûr, cette intégration peut avoir des répercutions externes, notamment dans les relations avec les partenaires chargés de la livraison des marchandises. L'intégration du SI est une condition nécessaire pour supporter l'intégration de sa chaîne logistique (Bensaou et Venkatraman, 1995 ; Truman, 2000). Cependant, les enjeux et les éventuels obstacles à surmonter se situent majoritairement en interne. 
L'intégration des SI, qu'elle soit interne ou externe, est source de performance en matière de coût, de qualité, et de profits (Maiga et al., 2015). Une explication possible du surcroit de performance imputable à l'intégration des SI réside dans le fait que cette intégration constitue une ressource à même de créer un avantage concurrentiel.

Il est crucial pour un distributeur omnicanal d'utiliser de manière effective les TI en intégrant les différentes fonctions de manière à ce que la cohérence et le flux des informations relatives aux clients, aux commandes et aux inventaires de stocks puissent être assurés (Cappiello et al., 2003 ; Markus, 2000 ; Vickery et al., 2003).

De même, le succès de l'intégration des canaux repose sur la capacité des distributeurs à intégrer en temps réel les informations relatives à l'ensemble des canaux, dans un unique SI. Ces informations peuvent concerner les clients (achats ou contact à travers les différents canaux offerts), les ventes réalisées par canaux et points de vente, les parcours clients, l'état des stocks, etc. En ce qui concerne les stocks, la capacité à intégrer au niveau du SI les inventaires des différents stocks centralisés, décentralisés et en magasin est un facteur clé de succès (Hübner et al., 2016).

Oh et al. (2012), identifient six facilitateurs d'intégration dans un contexte omnicanal, dont quatre impliquent directement le SI : la gestion intégrée de l'information relative aux transactions, une gestion intégrée de l'information relative aux produits et aux prix, un accès intégré à l'information, quel que soit le point de contact, un processus de traitement des commandes intégré, un service aux clients intégré et, pour terminer, une promotion des canaux intégrée.

Dans une étude empirique portant sur trois cas, Lewis et al. (2014) soulignent l'existence de barrières à l'intégration. Ils mettent au jour notamment des obstacles liés aux infrastructures informatiques et logistiques. L'existence de ces barrières à l'intégration peut expliquer les réticences de la direction. Ainsi, il est parfois difficile de convaincre l'équipe dirigeante de la nécessité de réaliser des investissements, alors que les ventes additionnelles résultant de l'intégration des canaux sont encore limitées. Le coût de la technologie est également évoqué par Piotrovicz et ses co-auteurs (2014) qui se demandent dans quelle mesure une expérience améliorée peut influencer les ventes et justifier une hausse des investissements.

\subsubsection{La gestion de la logistique \& de la supply chain}

Le management de la supply chain est un concept dont les contours sont variables : d'une approche purement intra-organisationnelle (locale fermée) à une approche globale (intégrant l'ensemble des parties prenantes), en passant par une approche locale ouverte (Fabbe-Costes, 2002b). Dans cette recherche, nous retenons, comme niveau d'analyse de la supply chain, l'approche locale ouverte, au sens de Fabbe-costes (2002b : p.31), dans lequel « la firme est principalement centrée sur la logistique intra-organisationnelle mais prend en compte les interfaces avec ses partenaires directs en amont et en aval ». Certains distributeurs vont au-delà, adoptant une approche globale, mais l'intégration des canaux présente des défis importants en matière de logistique intra-organisationnelle (entrepôts centralisés, stocks en magasins) et d'interfaces directes, notamment vers l'aval, vis-à-vis des clients.

La logistique est définie comme une technologie de pilotage optimal des flux physiques grâce à des flux d'information associés (Tixier et al., 1996). Duong et Paché (2015 : p. 56) affirment que « la maîtrise logistique se présente désormais comme une source d'avantage concurrentiel pour les entreprises ». Ils soulignent cependant que les ressources logistiques qui en sont le vecteur sont plus rarement évoquées, alors que, selon eux, l'acquisition d'un avantage 
concurrentiel, dans ce domaine, se fait «au prix d'une intégration performante de ressources internes et externes ».

L'intégration des canaux se traduit par des conséquences logistiques : «in terms of logistical operations, multi-channel integration consists of coordinating supply chains associated with different channels to create common "knots" (e.g. warehouses and platforms), so as to create economies of scale, as well as differentiating operations, particularly final delivery where customers have specific expectations." (Jeanpert et Paché, 2016 : p. 15).

La stratégie d'intégration des canaux renvoie à la question de l'intégration de l'assortiment d'une enseigne : jusqu'à quel point les assortiments doivent-ils être identiques ? La question de la composition de l'assortiment a été abordée récemment par Emrich et al. (2015) qui montrent que l'intégration totale - c'est-à-dire une similarité parfaite des assortiments proposés par chaque canal - est supérieure à une intégration partielle. L'asymétrie introduit un biais négatif. Le canal dont l'assortiment est le plus large sert de point de référence et le consommateur perçoit comme une perte, le fait qu'un autre canal offre un assortiment plus restreint.

Le système logistique doit être intégré et adapté à des livraisons individualisées (Lewis et al., 2014). Un mix entre solutions traditionnelles et en ligne est nécessaire (Piotrowicz et al., 2014). Le commerce omnicanal implique de repenser non seulement la logistique de livraison, mais également la logistique des retours (Hübner et al., 2016). En effet, les consommateurs sont demandeurs de dispositifs simples et pratiques pour retourner les marchandises commandées en ligne, lorsqu'elles ne conviennent pas. Ces questions sont au cœur des préoccupations des distributeurs interrogés par Hübner et al. (2016). Ils évoquent cinq priorités : 1) développer et optimiser les modes de livraison au client, 2) raccourcir la livraison, 3) fournir aux clients des informations sur la disponibilité et sur les délais de livraison pour tous les canaux, 4) optimiser les processus entre l'entrepôt centralisé et les magasins et 5) la consolidation des stocks destinés aux différents canaux.

\subsubsection{Les Ressources Humaines}

Certaines capacités du personnel constituent une compétence clé de l'intégration des canaux (Oh et al., 2012). Ces cross-channel human resource capabilities sont définies par Payne et Frow (2004) comme "frontline employee's awareness, business skills, and technical knowledge about the entire set of service offerings across all of the channels. Cette compétence repose sur la connaissance des vendeurs. Concrètement, les vendeurs en magasin doivent connaître toutes les options offertes au client telles que le click-\&-collect ou l'e-réservation qui autorise, pour la seconde, le paiement en magasin alors que la réservation est effectuée en ligne. Un niveau élevé de ces capacités est bénéfique: l'effet de l'intégration des SI sur la performance du distributeur est d'autant plus important (Oh et al., 2012). Les capacités des RH jouent également un rôle modérateur entre l'intégration des SI et la capacité à innover du distributeur (Oh et al., 2012). L'accompagnement des vendeurs en magasins, sous forme d'information et de formation aux objectifs et dispositifs relevant de l'omnicanal, est donc un élément clé du succès du passage du multicanal à l'omnicanal.

L'arrivée de la technologie dans les points de vente induit de nombreux changements. Faute d'un accompagnement approprié - notamment d'actions de formation - ces changements sont susceptibles d'engendrer des phénomènes de résistance de la part des vendeurs. Les chercheurs en SI se sont depuis longtemps intéressés aux phénomènes de résistance/acceptation de la technologie sur le lieu de travail. Le modèle d'acceptation de la technologie ou TAM (Davis, 1989) est l'un des premiers cadres théoriques à souligner le rôle crucial, pour l'adoption des TI par les salariés, de leur utilité et de leur facilité d'utilisation perçues. Par la suite, ce modèle a 
été complété, intégrant la norme subjective, et il a été montré que les variables influençant l'attitude diffèrent selon que l'adoption de la nouvelle technologie se fait sur la base du volontariat ou est imposée (Venkatesh et al., 2003). Dans le contexte omnicanal, Spreer et Rauschnabel (2016) identifient six antécédents de la résistance à l'adoption d'assistants mobiles pour les vendeurs (AMV) : un état de tension (crainte de commettre des erreurs devant le client), l'incongruence (entre l'image de la marque et l'image des AMV), la perte de pouvoir (la peur d'être remplacé par la technologie), la détérioration de la relation (impolitesse, méfiance vis-àvis des informations transmises), les imperfections opérationnelles (dysfonctionnements) et les investissements requis (notamment le temps nécessaire à la familiarisation).

L'accompagnement des $\mathrm{RH}$, tout au long du processus d'intégration, est nécessaire pour en faire une ressource essentielle de la transformation digitale des distributeurs et limiter la résistance au changement. Il est crucial d'informer les vendeurs des changements, de les former aux technologies digitales implantées dans les magasins et de les accompagner tout au long du changement (Spreer et Rauschnabel, 2016). Les changements occasionnés par la digitalisation du point de vente nécessitent la prise en compte du facteur humain durant les trois phases (premier stade, stade intermédiaire et stade avancé de mise en œuvre) identifiées par Lewis et al. (2014), alors qu'ils étudiaient les effets pour trois distributeurs de l'ajout du canal Internet. Dès la première phase, il s'agit de recruter ou de former le personnel (Lewis et al., 2014, p.58). En phase intermédiaire, il s'agit de gérer les conflits entre les personnels des différents canaux. En fin de processus, des problèmes liés à la culture et à l'engagement du personnel doivent être surmontés. Lewis et al. (2014) observent que l'intégration des canaux s'est traduite par un changement de culture dans les trois cas étudiés alors que la littérature antérieure n'y faisait pas référence.

\subsection{La théorie des ressources : cadre théorique de l'intégration des canaux}

La théorie des ressources, souvent mentionnée dans son acception anglo-saxonne comme la Resource-based view (RBV), considère que l'origine de la croissance des entreprises provient de ressources productives tangibles et intangibles (Penrose, 1959). Ce cadre a été complété par Wernerfelt (1984): alors qu'il s'interrogeait sur ce qui fait qu'une entreprise est plus performante que d'autres sur une longue période, Wernerfelt trouve la réponse non pas dans la position de la firme sur le marché ni dans les produits qu'elle développe. La performance durable provient d'un facteur interne à la firme: ses ressources, qu'il définit comme un ensemble d'actifs tangibles et intangibles liés de façon semi-permanente à l'entreprise. Les ressources font référence au «stock de facteurs disponibles, possédés ou contrôlés par une firme » (Amit et Schoemaker, 1993, p. 35). La RBV est centrée sur les ressources internes en tant que moyen d'obtenir un avantage concurrentiel. Pour que ces ressources soutiennent un avantage concurrentiel, elles doivent être «valuable, rare, imperfectly imitable and non substituable », d'où l'acronyme VRIN proposé par Barney (1991). La RBV suggère qu'une organisation doit développer des compétences centrales qui lui permettront de surpasser les concurrents en faisant les choses différemment (Prahalad et Hamel, 1990). Les compétences sont le mode d'organisation des ressources (Arrègle, 1995). Elles renvoient aux «capacités de l'entreprise à démultiplier ses ressources en les combinant » (Prahalad et Hamel, 1990, p. 26).

L'approche RBV s'est révélée particulièrement pertinente pour comprendre l'aptitude des firmes à intégrer des ressources en vue de produire des services et à s'engager dans des activités innovantes (Goldstein et al., 2002 ; Sirmon et al., 2007). En s'appuyant sur ce cadre théorique, Duong et Paché (2015) identifient cinq ressources clés pour le management stratégique de la logistique: les ressources technologiques, physiques, organisationnelles, relationnelles et d'expertise. Certaines de ces catégories se recoupent avec la définition que donne Reix et al. (2016) des SI. Ils énumèrent, en effet, le matériel, les logiciels, le personnel, les données, et les procédures. 
Reconsidérer l'intégration des canaux à l'aune de la théorie des ressources suggère que la combinaison de ressources internes est essentielle à l'acquisition d'une compétence centrale à même de soutenir un avantage concurrentiel. En intégrant les activités des différents canaux, les distributeurs peuvent rendre plus difficile l'imitation du fait de l'interconnexion des ressources intégrées. Il sera plus difficile pour les concurrents d'identifier les facteurs de succès. La pertinence de ce cadre théorique dans le cas de l'intégration des canaux de distribution physiques et digitaux a été démontrée par Oh et al. (2012). Ces auteurs montrent que les distributeurs intégrés sont plus efficients pour délivrer l'offre actuelle, mais ils présentent, en outre, une capacité d'innovation supérieure. Plus récemment, Luo et al. (2016) valident, dans un contexte de commerce omnicanal, une relation positive entre les ressources relevant des TI et les capacités inter-canal développées par les distributeurs.

Si la littérature montre que la théorie des ressources est adaptée au contexte d'intégration des canaux, il n'existe pas à notre connaissance, de recherche proposant une grille de lecture prenant en considération l'ensemble des ressources concernées par la stratégie d'intégration des canaux. Nous reprenons cette théorie et y intégrons la grille de lecture en cinq dimensions proposée par Duong et Paché (2015) en l'adaptant au contexte de l'intégration des canaux de distribution et en l'illustrant à l'aide d'exemples dans ce contexte (Tableau 1).

Pour chaque catégorie de ressources, quelques précisions sont nécessaires pour comprendre l'adaptation au contexte de l'omnicanal.

- Du point de vue des ressources technologiques, l'intégration des bases de données clients et stocks, en temps réel, est essentielle car elle contribue à mieux connaître les parcours inter-canaux des clients et à optimiser la logistique de sortie. Les technologies liées à Internet, notamment mobiles, et la Technologie Digitale en Magasin (TDM) constituent également des ressources cruciales car elles facilitent l'intégration des canaux.

- Du point de vue des ressources organisationnelles, les catégories évoquées par Duong et Paché (2015) sont aisément transposables au contexte de l'intégration des canaux : une culture d'entreprise facilitant l'appropriation des nouvelles technologies par le personnel, la volonté stratégique et l'implication de la direction, et d'un point de vue plus opérationnel, le déploiement de processus commerciaux contribuant à l'intégration ainsi que la coordination intra-organisationnelles entre les activités du web et les magasins.

- En ce qui concerne les ressources physiques, les magasins (en tant que point de vente, point de retrait ou de retour de marchandises et point d'entreposage) constituent une ressource qui est au cœur du dispositif omnicanal. A cette ressource s'ajoutent les surfaces d'entreposage centralisées (entrepôts destinés au e-commerce, au réapprovisionnement des magasins, etc.).

- L'intégration des canaux se traduit par un changement de perspective relationnelle entre canaux. Des canaux qui pouvaient jusque-là être perçus comme concurrents (magasin Internet) doivent collaborer. La capacité à instaurer une relation de confiance et coordonner intelligemment les opérations logistiques entre canaux constituent les ressources relationnelles clés.

- Enfin, le succès de l'intégration des canaux repose sur une dernière catégorie de ressources, les ressources d'expertise qui relèvent essentiellement des RH, avec (1) les mesures de formation aux nouvelles technologies et aux enjeux de l'intégration des canaux et (2) les connaissances individuelles, notamment le degré de familiarisation aux spécificités du commerce connecté et à la maîtrise des nouveaux outils. 
Conformément à l'objectif de cet article, la réflexion autour des ressources nécessaires au déploiement d'une stratégie omnicanal est structurée selon les dimensions de la grille d'analyse de Duong et Paché (2015) qui a été adaptée au contexte de l'intégration des canaux. 


\begin{tabular}{|c|c|c|c|c|c|}
\hline & Ressources technologiques & Ressources organisationnelles & Ressources physiques & Ressources relationnelles & Ressources d'expertise \\
\hline $\begin{array}{l}\text { Définition } \\
\text { adaptée au } \\
\text { contexte de } \\
\text { l'intégration } \\
\text { des canaux de } \\
\text { distribution }\end{array}$ & $\begin{array}{l}\text { Ressources qui permettent aux } \\
\text { entreprises d'acquérir, de traiter et } \\
\text { de transmettre des informations } \\
\text { pour concrétiser des prises de } \\
\text { décision efficaces. } \\
\text { Ces ressources nécessitent un } \\
\text { système d'information intégré. }\end{array}$ & $\begin{array}{l}\text { Compétences des entreprises } \\
\text { en matière : de développement } \\
\text { des systèmes, de politiques et } \\
\text { de processus commerciaux... }\end{array}$ & $\begin{array}{l}\text { Éléments infrastructurels } \\
\text { nécessaires pour assurer le } \\
\text { fonctionnement efficace et } \\
\text { efficient de la chaîne logistique. } \\
\text { Ressources indispensables pour } \\
\text { assurer la livraison de produits } \\
\text { et services aux clients quand ils } \\
\text { le souhaitent, là où ils le } \\
\text { souhaitent et de la façon dont } \\
\text { ils le souhaitent. }\end{array}$ & $\begin{array}{l}\text { Capacité d'une entreprise à } \\
\text { construire des relations } \\
\text { étroites avec ses partenaires } \\
\text { clés à travers la collaboration } \\
\text { et la confiance mutuelle en } \\
\text { vue de se coordonner } \\
\text { efficacement, de partager des } \\
\text { informations pertinentes et de } \\
\text { satisfaire les demandes de } \\
\text { leurs clients. }\end{array}$ & $\begin{array}{l}\text { Capacité des entreprises à } \\
\text { développer les compétences } \\
\text { humaines et à intégrer des } \\
\text { équipes munies des } \\
\text { connaissances et expériences } \\
\text { techniques indispensables. }\end{array}$ \\
\hline $\begin{array}{l}\text { Illustration } \\
\text { dans le cadre } \\
\text { de } \\
\text { l'intégration } \\
\text { des canaux de } \\
\text { distribution }\end{array}$ & $\begin{array}{l}\text { Bases de données relatives aux } \\
\text { stocks, aux clients selon les canaux } \\
\text { Technologies Internet (interface } \\
\text { web : site web, applications, etc.) } \\
\text { Technologie digitale en magasin : } \\
\text { - bornes, écran tactile, miroir } \\
\text { connecté, etc. } \\
\text { - Équipement des vendeurs } \\
\text { (smartphone, tablette, etc.) }\end{array}$ & $\begin{array}{l}\text { Culture d'entreprise } \\
\text { compatible avec la culture web } \\
\text { Politiques (décisions } \\
\text { stratégiques favorisant } \\
\text { l'intégration des canaux et } \\
\text { réduisant la concurrence entre } \\
\text { canaux) et implication des } \\
\text { équipes dirigeantes (mise en } \\
\text { place de groupes projets, etc.) } \\
\text { Processus commerciaux } \\
\text { Coordination des activités } \\
\text { intra-organisationnelles (site } \\
\text { web - magasins) }\end{array}$ & $\begin{array}{l}\text { Surface d'entreposage } \\
\text { (entrepôts centralisés et/ou } \\
\text { stocks en magasin) } \\
\text { Nombre de points de vente } \\
\text { Aménagement des points de } \\
\text { vente (point de retrait pour le } \\
\text { click-\&-collect, par exemple) }\end{array}$ & $\begin{array}{l}\text { Relation de confiance entre } \\
\text { les canaux } \\
\text { Coordination des opérations } \\
\text { logistiques mettant en jeu les } \\
\text { entrepôts web centralisés et } \\
\text { des stocks décentralisés en } \\
\text { magasin (livraison client final } \\
\text { / réassortiment des magasins) }\end{array}$ & $\begin{array}{l}\text { Mesures de formation aux } \\
\text { nouvelles technologies, aux } \\
\text { enjeux de l'intégration des } \\
\text { canaux, etc. } \\
\text { Connaissances individuelles : } \\
\text { familiarisation avec les } \\
\text { spécificités du commerce } \\
\text { connecté, maîtrise des } \\
\text { nouveaux outils, etc. }\end{array}$ \\
\hline
\end{tabular}

Tableau 1 - Les cinq ressources clés pour une intégration réussie des canaux de distribution (adapté de Duong et Paché, 2015) 


\section{Méthodologie}

Afin d'identifier les ressources mobilisées lors des stratégies d'intégration mises en œuvre par les entreprises, une étude qualitative exploratoire reposant sur la méthode des cas a été réalisée. La posture adoptée pour cette recherche relève des épistémologies constructivistes lesquelles reposent sur l'hypothèse d'une « réalité connaissable qui, pour être connue doit pouvoir être cognitivement construite ou reconstruite intentionnellement par un observateur-modélisateur » (Le Moigne, 2012, p. 45). En raison du manque de connaissance sur l'objet de la recherche (les ressources nécessaires au succès des stratégies d'intégration des canaux), les méthodes de recherche qualitatives sont, a priori, plus appropriées et davantage cohérentes avec le positionnement épistémologique retenu, que les approches hypothético-déductives préconisées pour les épistémologies positivistes ou post-positivistes.

La méthode des cas est fréquemment utilisée pour étudier le phénomène de transition d'un système de distribution en silos, vers un système intégré (Cao, 2014 ; Lewis et al., 2014 ; Saghiri et al., 2017). La méthode des cas correspond à une enquête empirique qui examine un phénomène contemporain au sein de son contexte réel (Yin, 2014). Les entreprises ont été sélectionnées selon des critères recommandés pour une telle méthodologie (HLady-Rispal, 2002) (Tableau 2).

\begin{tabular}{|c|c|c|}
\hline $\begin{array}{l}\text { Critères de } \\
\text { sélection }\end{array}$ & Définition & Respect du critère \\
\hline $\begin{array}{l}\text { Représentativité } \\
\text { théorique }\end{array}$ & $\begin{array}{l}\text { Homogénéité des cas du } \\
\text { point de vue de la question } \\
\text { à étudier }\end{array}$ & $\begin{array}{l}\text { - Disposer de magasins et d'un site de vente en } \\
\text { ligne. } \\
\text { - Processus d'intégration des canaux engagé. }\end{array}$ \\
\hline Variété & $\begin{array}{l}\text { Recherche de } \\
\text { différents les uns } \\
\text { autres }\end{array}$ & $\begin{array}{l}\text { - Trajectoires variées : Cultura, Nicolas, De } \\
\text { Serres, Aldo et la SAQ étaient initialement des } \\
\text { enseignes du monde physique et ont ensuite } \\
\text { développé un site d'e-commerce, alors que } \\
\text { Spartoo a un parcours inverse (du e-commerce } \\
\text { vers les boutiques). } \\
\text { - Secteurs d'activité variés : vins \& spiritueux, } \\
\text { chaussures \& accessoires et culture \& loisirs } \\
\text { créatifs. } \\
\text { - Ancienneté : de la plus ancienne Nicolas (1822) } \\
\text { à la plus récente, Spartoo (2006). } \\
\text { - Différents stades d'intégration des canaux } \\
\text { physiques et Internet : des balbutiements avec } \\
\text { De Serres, à l'intégration quasi-totale pour } \\
\text { Cultura et Spartoo, en passant par un stade } \\
\text { intermédiaire, avec Nicolas. } \\
\text { - Nationalité : française et canadienne. }\end{array}$ \\
\hline $\begin{array}{l}\text { Potentiel de } \\
\text { découverte }\end{array}$ & $\begin{array}{l}\text { Richesse des données sur } \\
\text { le phénomène de l'étude }\end{array}$ & $\begin{array}{l}\text { - Plusieurs entretiens pour chaque enseigne. } \\
\text { - Données primaires croisées avec les } \\
\text { informations disponibles dans la presse } \\
\text { managériale. } \\
\text { - } \\
\begin{array}{l}\text { Données d'observation issues de visites en } \\
\text { boutiques et sur les sites web. }\end{array}\end{array}$ \\
\hline
\end{tabular}

Tableau 2 - Critères de sélection des cas 
Les stratégies pour la mise en place de l'omnicanal de distributeurs français et canadiens sont comparées dans trois secteurs : vins \& spiritueux, chaussures $\&$ accessoires et culture $\&$ loisirs créatifs. Ces secteurs ont été choisis car ils représentent une part significative des produits achetés en ligne par les consommateurs $\left(50 \%\left(\right.\right.$ France $\left.^{2}\right) / 43 \%\left(\mathrm{Canada}^{3}\right)$ pour les produits culturels, 39\% (France) / 24\% (Canada) pour les chaussures et 20\% (France) / 13\% (Canada) pour les produits alimentaires dont le vin) et la complémentarité magasin / site Internet est importante pour ces types de produits. Des paires ont été constituées afin de disposer d'une enseigne pour chaque catégorie de produit dans les deux pays. Ainsi, six cas d'entreprises ont été retenus : www.nicolas.com, www.cultura.com et www.spartoo.com pour la France et www.saq.com, www.deserres.com et www.aldoshoes.com/ca, pour le Canada. Le tableau 3 présente les caractéristiques des distributeurs ayant participé à l'étude, le nombre d'entretiens réalisés ainsi que le profil des personnes interviewées.

Dix entretiens semi-directifs d'une durée moyenne d'une heure ont été conduits auprès d'acteurs identifiés comme stratégiques de l'intégration des canaux chez les six distributeurs étudiés (PDG, responsable marketing, responsable des opérations). Plusieurs entretiens ont été réalisés par distributeur lorsque cela était possible. Les entretiens ont été conduits selon un guide disponible sur demande. Les thèmes suivants ont été abordés : principales étapes dans l'histoire du distributeur et ses chiffres clés, évolution du distributeur du multicanal vers l'omnicanal, impacts de l'omnicanal sur l'organisation, le SI, les vendeurs, l'offre, l'aménagement des magasins, la logistique, etc. Outre le corpus issu des entretiens, les sites ecommerce des distributeurs ont été analysés et une visite des points de vente a été réalisée. Enfin, ces données primaires ont été complétées par des données secondaires issues de la presse managériale (LSA, Journal du Net, etc.).

Les entretiens ont été réalisés en face-à-face et ont été enregistrés. Ils ont été retranscrits et analysés à l'aide du logiciel NVivo 12 qui permet une rigueur dans le codage des données. Afin d'obtenir une bonne fiabilité du codage, un entretien a d'abord été codé par deux codeurs et le taux d'accord entre les codeurs était de 95\% après résolution des divergences, le codage s'est poursuivi selon la même méthode. Le taux d'accord final était comparable à celui précédemment obtenu. La grille d'analyse développée par Duong et Paché (2015) préalablement adaptée à l'intégration des canaux de distribution a été retenue pour l'analyse des résultats. Les données ont été codées selon les cinq catégories proposées par Duong et Paché (2015). Une analyse thématique inter-distributeur a été réalisée en fonction de ces cinq catégories et des sous-thèmes mentionnés dans le tableau 1 ci-dessus.

\section{Résultats : L'intégration des canaux dans la pratique}

L'analyse de contenu réalisée selon les thématiques recensées dans la grille de Duong et Paché (2015), permet d'illustrer point par point, les ressources qui sous-tendent l'intégration des canaux. L'analyse réalisée avec le logiciel Nvivo permet d'obtenir la fréquence avec laquelle les cinq catégories de ressources sont évoquées par les personnes interviewées. Globalement, 241 éléments de texte ont été codés comme évoquant une des ressources. La ressource organisationnelle est celle qui présente la plus forte occurrence (96 occurrences, soit $40 \%$ ). Les ressources technologiques suivent avec 70 occurrences $(29 \%)$.

\footnotetext{
${ }^{2}$ Baromètre FEVAD/CSA, janvier 2019

${ }^{3}$ Statista global consumer survey, 2018
} 


\begin{tabular}{|c|c|c|c|c|c|c|c|}
\hline & $\begin{array}{l}\text { Date et } \\
\text { lieu de } \\
\text { création }\end{array}$ & $\begin{array}{c}\text { Produits } \\
\text { commercialisés }\end{array}$ & Chiffre d'affaires & $\begin{array}{l}\text { Nombre de } \\
\text { magasins }\end{array}$ & Parcours & $\begin{array}{c}\text { Type de réseau/ statut } \\
\text { des boutiques }\end{array}$ & Pays \\
\hline $\begin{array}{l}\text { Aldo (1 entretien) } \\
\text { - Directeur des opérations } \\
\text { e-commerce }\end{array}$ & $\begin{array}{c}1972 \\
\text { Montréal }\end{array}$ & $\begin{array}{l}\text { Chaussures \& } \\
\text { accessoires }\end{array}$ & $\begin{array}{l}\text { 1,6 milliards de } \\
\$ \mathrm{CAD}^{*} \text {, dont } 15 \% \\
\text { en ligne }(2015)\end{array}$ & $\begin{array}{l}\text { Plus de } 3000 \\
\text { magasins }\end{array}$ & $\begin{array}{c}\text { Magasins } \rightarrow \\
\text { Click \& mortar } \\
(2005) \\
\end{array}$ & $\begin{array}{c}\text { Succursales (Amérique } \\
\text { du Nord, UK) / } \\
\text { franchises ailleurs } \\
\end{array}$ & $\begin{array}{l}\text { Canada } \\
95 \text { pays }\end{array}$ \\
\hline $\begin{array}{l}\text { SAQ (2 entretiens) } \\
\text { - DG } \\
\text { - VP finance }\end{array}$ & $\begin{array}{c}1921 \\
\text { Montréal }\end{array}$ & $\begin{array}{c}\text { Vin \& } \\
\text { Spiritueux }\end{array}$ & $\begin{array}{c}>3 \text { milliards de } \\
\$ C A D \text {, dont } 30 \\
\text { millions pour le site } \\
\text { Internet }\end{array}$ & 400 magasins & $\begin{array}{l}\text { Magasins } \rightarrow \\
\text { Click \& mortar } \\
\quad \text { (1999) }\end{array}$ & Succursales & $\begin{array}{l}\text { Québec / } \\
\text { Canada }\end{array}$ \\
\hline $\begin{array}{l}\text { De Serres } \\
\text { (1 entretien) } \\
\text { - PDG }\end{array}$ & $\begin{array}{c}1908 \\
\text { Montréal }\end{array}$ & $\begin{array}{l}\text { Beaux-Arts \& } \\
\text { loisirs créatifs }\end{array}$ & $\begin{array}{c}\text { N.C. } \\
\text { Internet représente } \\
5 \% \text { des ventes } \\
\text { totales }\end{array}$ & $\begin{array}{c}35 \text { magasins } \\
\text { dont près de } \\
\text { la moitié au } \\
\text { Québec }\end{array}$ & $\begin{array}{l}\text { Magasins } \rightarrow \\
\text { Click \& mortar } \\
\quad(2012)\end{array}$ & Succursales & Canada \\
\hline $\begin{array}{l}\text { Cultura (3 entretiens) } \\
\text { - Directeur du Commerce } \\
\text { Connecté } \\
\text { - Directeur de magasin } \\
\text { - Responsable marketing } \\
\text { digital - relation client }\end{array}$ & $\begin{array}{c}1998 \\
\text { La } \\
\text { Rochelle }\end{array}$ & $\begin{array}{c}\text { Culture \& } \\
\text { loisirs créatifs }\end{array}$ & $\begin{array}{l}700 \text { millions d' } € \\
(2017) \\
\text { CA e-commerce } 35 \\
\text { millions d' } €(5 \%) \\
\text { réaffecté aux } \\
\text { magasins } \\
\end{array}$ & 92 magasins & $\begin{array}{l}\text { Magasins } \rightarrow \\
\text { Click \& mortar } \\
\quad(2005)\end{array}$ & Succursales & France \\
\hline $\begin{array}{l}\text { Spartoo.com ( } 1 \text { entretien }) \\
\text { - Directeur Marketing }\end{array}$ & $\begin{array}{c}2006 \\
\text { Grenoble }\end{array}$ & $\begin{array}{l}\text { Chaussures } \\
\& \text { accessoires }\end{array}$ & $\begin{array}{l}140 \text { millions d'€, } \\
\text { dont magasins } 6 \text { à } 7 \\
\text { millions } \\
(2015)\end{array}$ & $\begin{array}{l}13 \text { magasins } \\
1 \text { ère ouverture } \\
\text { en } 2015\end{array}$ & $\begin{array}{l}\text { Pure player } \rightarrow \\
\text { Click \& mortar } \\
\quad(2015)\end{array}$ & Succursales & $\begin{array}{l}\text { France } \\
20 \text { pays }\end{array}$ \\
\hline $\begin{array}{l}\text { Nicolas (2 entretiens) } \\
\text { - Chef de projet web } \\
\text { - Responsable Marketing } \\
\text { Digital }\end{array}$ & $\begin{array}{l}1822 \\
\text { Paris }\end{array}$ & $\begin{array}{l}\text { Vins \& } \\
\text { Spiritueux }\end{array}$ & $\begin{array}{c}300 \text { millions d' } € \\
\text { (2016) }\end{array}$ & $\begin{array}{l}510 \text { magasins } \\
\text { en France } \\
38 \text { magasins à } \\
\text { l'étranger }\end{array}$ & $\begin{array}{l}\text { Magasins } \rightarrow \\
\text { Click \& mortar } \\
\quad(2000)\end{array}$ & $\begin{array}{c}\text { Mandataires gérants en } \\
\text { France } \\
14 \text { franchises } \\
\text { (objectif } 100 \text { en 2020) }\end{array}$ & $\begin{array}{l}\text { France } \\
6 \text { pays }\end{array}$ \\
\hline
\end{tabular}

$* \$ C A D=$ Dollars canadiens

\section{Tableau 3 - Les distributeurs ayant participé à l'étude}


Viennent ensuite les ressources relationnelles (30 occurrences, soit 12,5\%), puis les ressources d'expertise (23 occurrences, 9,5\%) et physiques (20 occurrences, 8,3\%). Une analyse plus fine, par ressources, est ensuite réalisée, en se focalisant sur les sous-catégories de ressources identifiées dans le tableau 1.

\subsection{Les ressources technologiques}

Les ressources technologiques font référence au SI. Le SI ressort comme une ressource essentielle (Spartoo, Cultura, SAQ). Le degré d'intégration du SI est une préoccupation partagée, mais celle-ci semble davantage maitrisée par Spartoo qui a pensé, dès le départ, son SI de manière à pouvoir intégrer les données du canal Internet et physique. Pour les autres distributeurs, le SI est, à plusieurs reprises, présenté comme un point bloquant: les caractéristiques actuelles du SI ne permettent pas d'intégrer totalement les données issues des différents canaux. "Chez Cultura, du point de vue du magasin et du point de vue du site Internet, les canaux ont été abolis. Il reste cependant à abolir les distinctions techniques (SI...). » Pour la SAQ: "Le principal blocage est un blocage technique lié à notre SI. La chaîne d'approvisionnement devra suivre et s'adapter à ces évolutions. »

Le verbatim relatif aux ressources technologiques (Tableau 4) porte sur les différentes composantes du SI : les bases de données, les technologies Internet, ainsi que les technologies digitales en magasin (TDM).

En ce qui concerne les bases de données, l'intégration des données relatives au stock est une ressource essentielle pour l'intégration des canaux. Cette ressource semble maîtrisée par Cultura, Aldo, Spartoo et la SAQ. Spartoo est probablement le distributeur qui est le plus avancé en matière de fluidité des données : "On a développé notre propre logiciel de caisse qui est interfacé avec notre base de données web. On peut donc tout savoir, comme l'augmentation du business web dans la zone de chalandise, le chiffre d'affaires additionnel en magasin des personnes qui se font livrer en click-\&-collect, la part de chiffre d'affaires d'un client entre le web et le magasin ». Cette ressource n'est pas encore maîtrisée pour De Serres et Nicolas. L'intégration des données clients constitue une autre ressource clé. Or, le niveau d'intégration en ce qui concerne les distributeurs étudiés est extrêmement variable. Le distributeur le plus avancé est la SAQ qui a déployé un système cross-canal de recommandations automatisées et personnalisées.

Les technologies liées à Internet sont également évoquées. La refonte du site web est la ressource la plus largement évoquée, mais certains distributeurs se démarquent grâce à une application (Aldo) ou par des projets de porte-monnaie et/ou de carte de fidélité électronique (Nicolas).

Les Technologies Digitales en Magasin (TDM) constituent également une ressource dans la mesure où elles permettent : de sauver une vente si le produit commandé en click-\&-collect ne convient pas, d'augmenter le panier moyen du fait d'un accès à l'intégralité de l'inventaire via la commande web en magasin et, enfin, d'améliorer l'expérience en magasin. Les distributeurs ont développé ou envisagent de développer des équipements destinés aux vendeurs (tablette, smartphone) et/ou des équipements utilisables par les clients en autonomie ou avec l'aide d'un vendeur (borne et miroir connecté). Ces équipements restent cependant «basiques » : aucun distributeur n'envisage de mettre en place des technologies intégrant la réalité augmentée, par exemple. Cultura, qui envisage de développer la géolocalisation indoor, se démarque quelque peu. 


\begin{tabular}{|c|c|}
\hline Facettes & Verbatim \\
\hline $\begin{array}{l}\text { Bases de } \\
\text { données : }\end{array}$ & $\begin{array}{l}\text { Aldo: «Le client qui commande en ligne (que ce soit depuis le magasin ou depuis son ordinateur / téléphone / tablette) a accès à l'ensemble de } \\
\text { l'inventaire de nos entrepôts et de nos magasins. " } \\
\text { De Serres: «Dans quelques mois, nous mettrons sur le site Internet l'inventaire du magasin pour vérifier la disponibilité du produit avant de se } \\
\text { rendre en magasin." }\end{array}$ \\
\hline Stocks & $\begin{array}{l}\text { Nicolas : "Si le client veut faire une e-réservation, il doit se connecter sur son compte et il doit à ce moment choisir un magasin. Si un produit n'est } \\
\text { pas en stock, le magasin va appeler la personne qui a e-réservé pour dire que finalement, le produit n'est pas disponible. " }\end{array}$ \\
\hline Clients & $\begin{array}{l}\text { Cultura: "Pour le moment, nous n’avons pas de référentiel client unique, permettant de suivre le client sur les différents canaux. " } \\
\text { Aldo : «Actuellement, nous avons toutes les données sur les clients mais elles ne sont pas encore connectées. Nous allons travailler sur ce point dans } \\
\text { les prochains mois. Cela passe par une modification de notre SI. » } \\
\text { SAQ : «En sachant ce que le client a commandé, le SI fait des recommandations de produits complémentaires que le vendeur peut proposer en } \\
\text { magasin. » [à propos du click-\&-collect] }\end{array}$ \\
\hline $\begin{array}{l}\text { Technologies } \\
\text { Internet (TI) }\end{array}$ & $\begin{array}{l}\text { De Serres : «La deuxième version du site a été lancée en octobre 2016. » } \\
\text { "L'application Aldo a officiellement été lancée au Canada et aux Etats-Unis. » } \\
\text { Nicolas : «La refonde [du site] a eu lieu en 2015. [...] On essaie de mettre en place le 'wallet', [...] une carte de fidélité dématérialisée comme dans } \\
\text { Fidme, mais dans le wallet, pour que les gens reçoivent des notifications push via l'intermédiaire du wallet et avec un système de géolocalisation. » }\end{array}$ \\
\hline $\begin{array}{l}\text { Technologie } \\
\text { digitale en } \\
\text { magasin (TDM) }\end{array}$ & $\begin{array}{l}\text { "Cultura a déployé des bornes dans ses magasins et a équipé ses conseillers de vente de terminaux numériques. [...] L'enseigne prévoit de développer } \\
\text { la géolocalisation indoor et une application mobile utilisable par le client notamment dans le magasin. » } \\
\text { Aldo : «Nos flagship stores sont équipés de tablettes et disposent même d'écrans connectés sur les murs ». } \\
\text { Spartoo : "Si les clients ne trouvent pas les chaussures qu'ils souhaitent en magasin, les vendeurs sont incités à pousser l'offre web (supplémentaire } \\
\text { ou complémentaire) grâce à une tablette ou à des écrans connectés ». } \\
\text { Nicolas : « les vitrines digitales en magasin [sont] en cours de déploiement. [...] Pas de projet d'équiper les cavistes de terminaux nomades. [...] Pas } \\
\text { de tablettes où la personne peut définir sa commande sur le web. » } \\
\text { "Pour le moment la SAQ ne déploie pas d'outils particuliers à destination des vendeurs. Des phases de test sont menées dans quelques succursales } \\
\text { afin de déterminer quelle interface serait optimale en magasin : écran interactif, tablette, smartphone... L'objectif est d'outiller nos employés. » }\end{array}$ \\
\hline
\end{tabular}

Tableau 4 - Les ressources technologiques 


\subsection{Les ressources organisationnelles}

Les ressources organisationnelles font référence aux compétences des entreprises en matière de développement de système, de politiques et de processus commerciaux. Quatre types de ressources organisationnelles sont identifiées : la culture d'entreprise, l'implication des équipes dirigeantes, la coordination intra- et inter-organisationnelle et les processus commerciaux (Tableau 5).

La culture click \& mortar est perçue, dans le discours, comme un frein à l'intégration des canaux, alors que la culture pure player jouerait le rôle de facilitateur de cette intégration. Il semblerait que la trajectoire allant du e-commerce vers l'omnicanal procure un avantage supérieur comparativement à la trajectoire amenant les distributeurs du commerce traditionnel vers l'omnicanal. L'ADN digital constituerait donc une ressource. L'argument est repris par Aldo et Nicolas. A ce point s'ajoute un autre handicap, celui de l'organisation du réseau de distributeur physique : le commerce associé ou franchisé, de même que le statut de gérantmandataire (Nicolas) seraient sources de freins à l'intégration des canaux car il serait plus difficile d'imposer la stratégie d'intégration aux responsables de magasins, qui ne bénéficient pas du statut de salarié.

L'implication des équipes dirigeantes ressort comme une ressource clé de l'intégration des canaux. Sans celle-ci, il est probable que l'intégration ne puisse pas avoir lieu. La décision d'intégrer les canaux doit être prise au plus haut niveau (entérinée par le Comité de Direction de la SAQ, par exemple) et se traduit par une modification de la structure de certains distributeurs (création d'une direction du commerce connecté chez Cultura).

Des processus commerciaux bien définis au préalable constituent également une ressource potentielle : profiter par exemple, d'une commande web en magasin pour ajouter un client dans la base de données et pouvoir le contacter par la suite (Cultura) : "La commande web en magasin permet aux conseillers de vente de s'approprier le canal Internet, de créer un fichier client pour pouvoir les solliciter éventuellement ultérieurement. 9 clients sur 10 qui passent par la commande web [en magasin], n'avaient pas de compte sur cultura.com... la commande web en magasin permet donc de recruter également de nouveaux clients pour le site. ». La SAQ met à profit le retrait en magasin pour proposer des produits additionnels. En revanche, toutes les enseignes n'ont pas pris conscience des enjeux du retrait en magasin (De Serres). Cultura a bien compris l'enjeu de ces processus commerciaux et cela ressort du dispositif de répartition de la valeur qui est mis en place. "Pour les commandes web passées en magasin par le conseiller de vente, le chiffre d'affaires (CA) est affecté au magasin. [...] Pour les commandes en ligne avec retrait en magasin (click-\&-collect) : le CA est affecté au magasin de retrait. [...] Pour les commandes en ligne passées dans la zone de chalandise d'un ou plusieurs magasins : le CA est affecté au(x) magasin(s) de la zone au prorata. [...] Pour les commandes en ligne passées hors d'une zone de chalandise d'un magasin : le CA est affecté à l'ensemble des magasins au prorata de leur CA des commandes en ligne. »

Enfin, une bonne coordination intra- et inter-organisationnelle, en ce qui concerne la préparation et l'expédition des commandes est considérée comme une ressource clé (Aldo, Spartoo, SAQ, De Serres). Des pistes d'amélioration sont envisagées sous la forme d'intégration de nouveaux partenaires (Uber, Instacart) chez Aldo. 


\begin{tabular}{|c|c|}
\hline Facettes & Verbatim \\
\hline $\begin{array}{c}\text { Culture } \\
\text { d'entreprise (CE) }\end{array}$ & $\begin{array}{l}\text { Aldo : "Etant donné que nous venons du monde physique, nous n'avons pas autant la culture « data » que peut avoir un pure player et cela va } \\
\text { nous prendre plus de temps. » } \\
\text { "Spartoo est une entreprise qui se veut agile, avec l'ADN d'un pure player et dont les valeurs de l'entreprise sont facilement partagées, de par sa } \\
\text { petite taille. » } \\
\text { "Nicolas, de par son mode d'organisation (gérants-mandataires et franchisés), ne peut pas avancer aussi vite qu'il le souhaiterait en matière de } \\
\text { vente en ligne. [...] Notre stratégie ce n'est pas d'être des pure player, comme Amazon. » }\end{array}$ \\
\hline $\begin{array}{c}\text { Politique et } \\
\text { implication des } \\
\text { équipes dirigeantes }\end{array}$ & $\begin{array}{l}\text { "En 2013, Cultura a créé une Direction du Commerce Connecté avec la volonté d'adopter une stratégie retail player. [...] Une 'core } \\
\text { transformation team' est en place afin de diffuser la philosophie du commerce connecté à l'intérieur de l'entreprise. " } \\
\text { De Serres: «Les magasins, de par notre organisation en magasins corporatifs, n'ont pas d'autre choix que d'évoluer vers le multicanal. » } \\
\text { "En 2013, Aldo développe et met en place une nouvelle stratégie commerciale multicanal afin d'interagir avec ses clients de façon fluide et } \\
\text { cohérente, quelle que soit la plateforme de vente ou de communication utilisée ». } \\
\text { Nicolas : "On a un chef de projet nommé en soutien, chef de projet e-livraison, livraison à domicile. » } \\
\text { SAQ : "Tous les éléments mentionnés vers l'omnicanal ont été entérinés par le comité de direction de la SAQ. Si ce n'était pas le cas, et le passé } \\
\text { a pu le montrer, il y a des chances pour que cela ne soit pas mis en oeuvre en réalité ». }\end{array}$ \\
\hline $\begin{array}{c}\text { Processus } \\
\text { commerciaux }\end{array}$ & $\begin{array}{l}\text { Cultura : «La commande web en magasin permet aux conseillers de vente en magasin de s'approprier le canal Internet, de créer un fichier client } \\
\text { pour pouvoir les solliciter éventuellement ultérieurement. } 9 \text { clients sur } 10 \text { qui passent par la commande web [en magasin], n'avaient pas de compte } \\
\text { sur Cultura.com... la commande web en magasin permet donc de recruter également de nouveaux clients pour le site. » } \\
\text { De Serres : « Lorsque nous mettrons en place le click-\&-collect, nous n'envisagerons pas d'effectuer la cueillette des produits en magasins. » } \\
\text { «Aldo ne croyait pas que ce mode de vente [en ligne] allait fonctionner pour des souliers. C'est pourquoi Aldo a mis en place un système de } \\
\text { fulfillment des commandes en ligne depuis ses magasins en fonction de la géo-proximité avec le client. [...] Le magasin qui a préparé la commande } \\
\text { et l'a faite expédiée reçoit lui l'équivalent d'une somme de } 7 \text { minutes de temps de travail par unité. » }\end{array}$ \\
\hline $\begin{array}{l}\text { Coordination intra- } \\
\text { / inter- } \\
\text { organisationnelle }\end{array}$ & $\begin{array}{l}\text { Aldo : "Nous sommes en train de faire une étude sur les profits que l'on a à avoir notre inventaire dans les magasins versus les profits que l'on a } \\
\text { à avoir notre inventaire dans un entrepôt et nous allons comparer cela avec le nombre d'annulation de commandes en ligne à cause des délais et } \\
\text { les coûts de livraison depuis notre entrepôt basé à Montréal pour toute l'Amérique du Nord. » } \\
\text { "Les commandes en click-\&-collect sont expédiées depuis l'entrepôt de SAQ.com à Montréal. Ce qui fait que les délais de livraison pour la } \\
\text { commande livrée en magasin (tout comme les commandes livrées à domicile) peuvent atteindre } 3 \text { à } 5 \text { jours. [...] Le programme " cliquer-acheter- } \\
\text { ramasser » a été mis en place pour supprimer les transferts inter-succursales qui étaient très couteux. » } \\
\text { De Serres : «La préparation et l'expédition des commandes en ligne sont gérées par un sous-traitant qui est rémunéré par un coût variable (en } \\
\text { fonction du nombre d'articles dans la commande). » } \\
\text { Aldo: «Nous voulons [...] développer les modes de livraison (via Uber, Instacart...). » }\end{array}$ \\
\hline
\end{tabular}

\section{Tableau 5 - Les ressources organisationnelles}




\subsection{Les ressources physiques}

Les ressources physiques sont des éléments d'infrastructure nécessaires au fonctionnement de la chaîne logistique. Ces ressources permettent d'assurer la livraison des produits aux clients quand ils le souhaitent, là où ils le souhaitent et de la façon dont ils le souhaitent. Trois catégories de ressources physiques nécessaires à l'intégration des canaux sont identifiées : les surfaces d'entreposage, le réseau de magasins et l'aménagement du point de vente (Tableau 6). Deux autres ressources sont ponctuellement évoquées: la capacité de traitement des commandes et les équipements de transport.

Les surfaces d'entreposage constituent une ressource cruciale, notamment le choix de leur emplacement. Il se trouve dans la région parisienne pour Cultura, en région lyonnaise pour Spartoo et dans la région de Montréal pour les trois distributeurs canadiens, SAQ, Aldo et De Serres. Des relocalisations, que l'on peut imaginer stratégiques, sont parfois opérées : Aldo a déplacé son entrepôt européen du Royaume Uni vers les Pays-Bas. Il arrive que le même entrepôt soit utilisé pour les réapprovisionnements des magasins et les commandes web (De Serres).

Le nombre de points de vente, qui varie d'une quinzaine (Spartoo) à 2000 (Aldo), est présenté comme une force, ce qui en fait une ressource car ce sont autant de points de contacts et d'occasions de contact qui peuvent être mis à profit si des processus systématiques de collecte d'information et/ou de recommandations existent et sont effectivement utilisés, comme cela a été évoqué pour Cultura. De plus, les magasins, outre leur fonction première de vente et la possibilité d'améliorer la connaissance client, constituent potentiellement des surfaces de stockage additionnelles lorsque la livraison depuis le magasin ou la livraison de magasin à magasin est envisagée. Aucune enseigne n'envisage de réduire le nombre de points de vente. Pour Spartoo, dont l'ADN est celui d'un pure player, l'ouverture de magasins était incontournable et l'enseigne envisageait de développer son réseau physique ${ }^{4}$.

L'aménagement du point de vente n'est, semble-t-il, pas perçu comme une ressource essentielle, sauf peut-être pour la SAQ qui envisage de réduire la surface des magasins. La nécessité de réaménager le point de vente est davantage perçue comme une conséquence de la Technologie Digitale en Magasin (Cultura).

\footnotetext{
${ }^{4}$ Après le rachat des chaussures André en 2018, certains magasins André avaient été transformés en points relais Spartoo et des corners Spartoo équipés d'écrans donnaient accès à une collection dédiée au sport. Déjà fragilisées avant la crise sanitaire, les chaussures André ont été placées en redressement judiciaire durant l'été 2020 et seront reprises par l'ancien dirigeant. https://www.lemonde.fr/economie/article/2020/04/01/1-enseigne-andre-placee-enredressement-judiciaire 6035232 3234.html ; https://www.lesechos.fr/industrie-services/conso-distribution/unsauveur-pour-les-chaussures-andre-1226932
} 


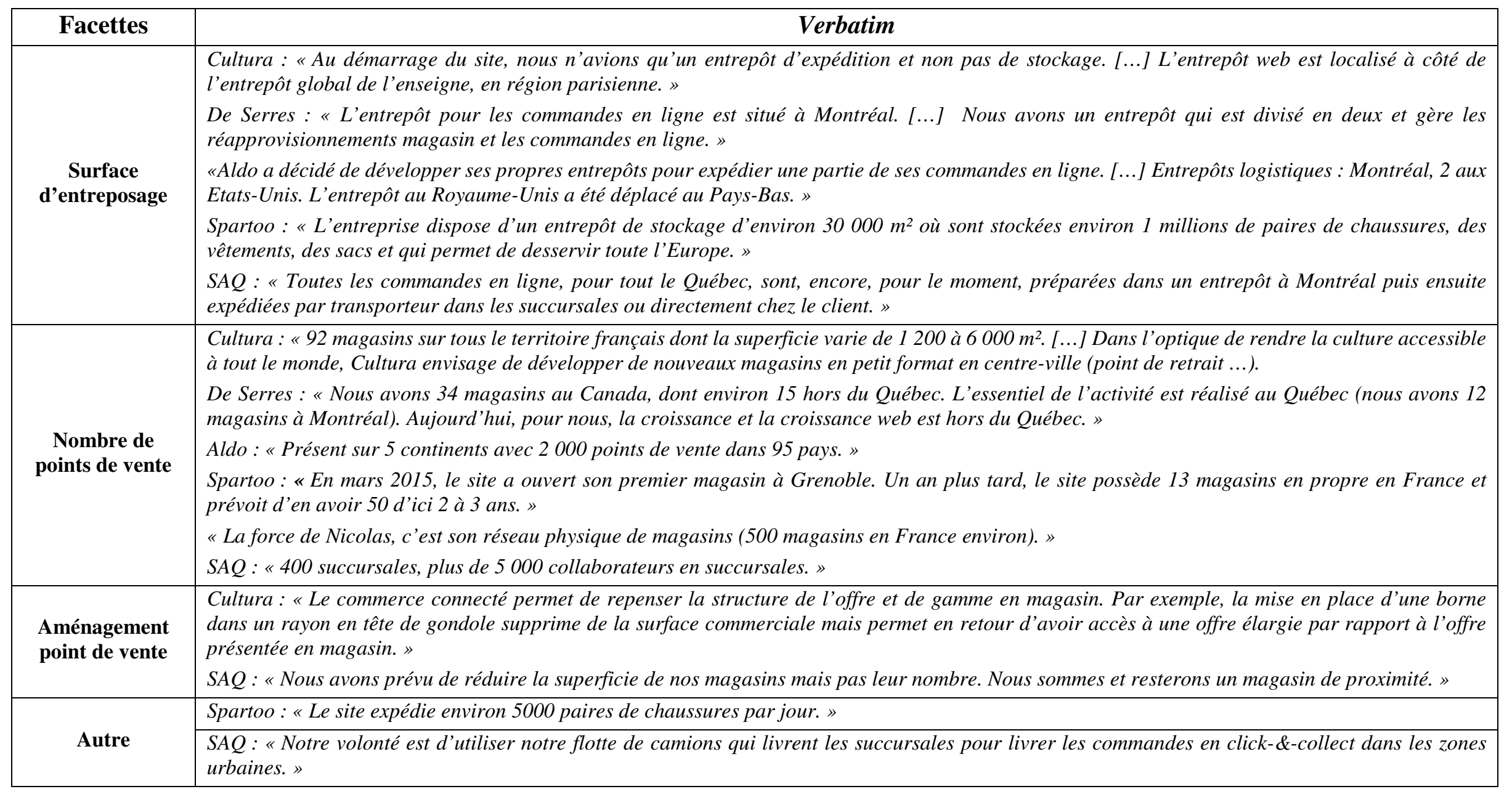

Tableau 6 - Les ressources physiques 


\subsection{Les ressources relationnelles}

Les ressources relationnelles font référence à la capacité d'une entreprise à construire des relations étroites avec ses partenaires clés à travers la collaboration et la confiance mutuelle en vue de se coordonner efficacement, de partager des informations pertinentes et de satisfaire les demandes de leurs clients. Deux catégories ressortent du verbatim : la confiance entre canaux et la coordination des opérations logistiques (Tableau 7). La question de l'échange d'information entre canaux est peu évoquée.

La confiance entre canaux physique et magasin constitue une ressource clé car elle facilite le déploiement de la stratégie d'intégration. A chaque nouvelle étape de l'intégration, la confiance peut être remise en question et céder la place à la méfiance ou aux tensions. La présence de termes négativement connotés dans le verbatim reflète davantage le manque de confiance plutôt que la confiance entre canaux : « concurrence », « cannibalisation », « tensions », «frictions », « réactions de méfiance », «menaces », « résistance», « réticents » et «frustrés ». La plupart des distributeurs (Cultura, Aldo, SAQ, Nicolas) ont connu, à un moment où à un autre, une perte de confiance, la vente en ligne étant perçue comme une menace. Cela s'est parfois traduit par des tensions et des phénomènes de résistance. La confiance ne se limite pas à une confiance entre canaux. Elle est intimement liée à la confiance entre les clients et les vendeurs. Deux distributeurs mettent l'accent sur cet aspect : Nicolas, pour lequel le caviste reste au cœur du système de distribution et Cultura, qui évoque la symétrie des attentions "une idée selon laquelle la qualité de la relation entre une entreprise et ses clients est égale à la qualité de la relation de cette entreprise avec ses propres collaborateurs».

Une coordination optimisée des opérations logistiques peut rééquilibrer la relation entre les canaux et constitue, en ce sens, une ressource. Il peut s'agir de ne pas réduire le magasin à un simple point relai et/ou à un lieu de stockage destiné à honorer les commandes en ligne. Il est crucial de faire en sorte que le temps des vendeurs ne soit pas trop absorbé par la préparation des commandes web, de leur laisser la possibilité de refuser la préparation des commandes, comme c'est le cas chez Aldo. Chez ce dernier, $90 \%$ des commandes en ligne étaient expédiées depuis les magasins, ce qui pouvait créer des tensions et de la «mauvaise volonté ». Aldo a fait évoluer ce modèle pour une meilleure répartition entre les expéditions depuis les entrepôts centralisés et depuis les magasins (50/50). Lorsque les points de vente sont en concurrence du fait de leur proximité et du statut juridique, il semble plus délicat d'envisager des livraisons de magasin à magasin. C'est une difficulté qui est évoquée par Nicolas. Avec ses 500 magasins sur le territoire français, il n'est pas rare que deux magasins se trouvent dans la même zone de chalandise. 


\begin{tabular}{|c|c|}
\hline Facettes & Verbatim \\
\hline $\begin{array}{c}\text { Relation de } \\
\text { confiance entre les } \\
\text { canaux }\end{array}$ & 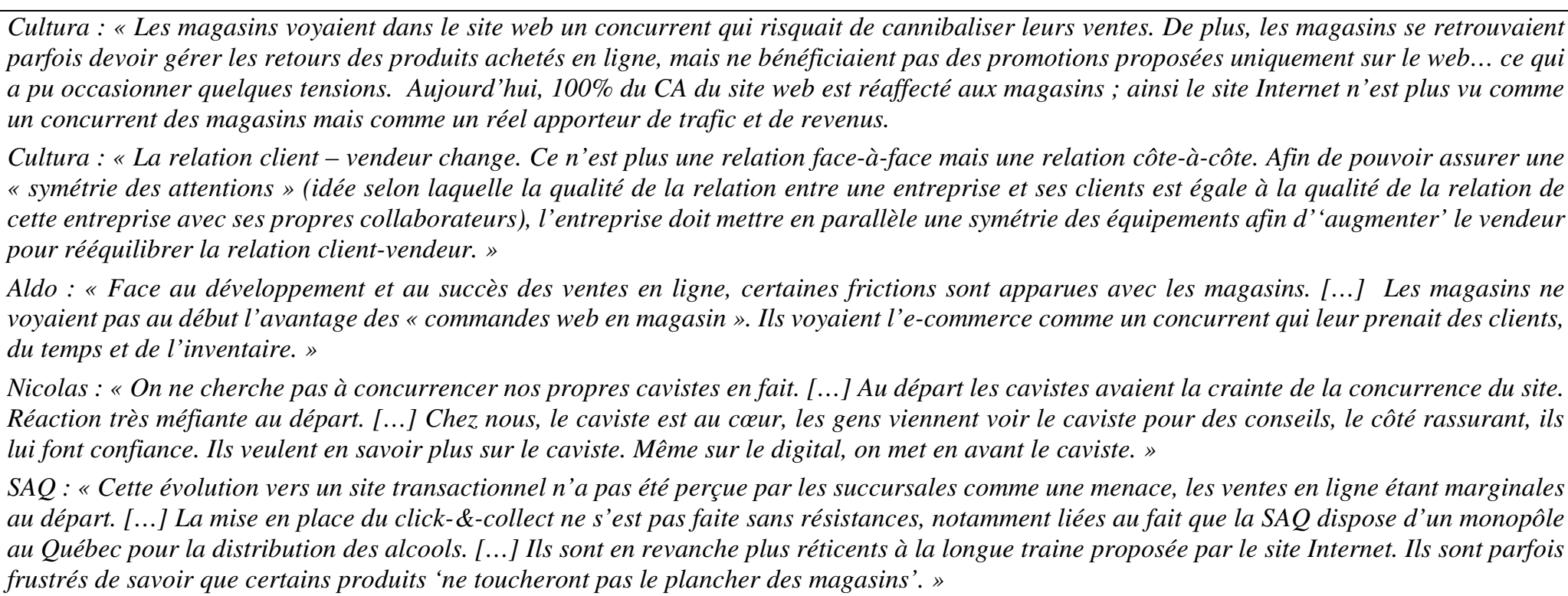 \\
\hline $\begin{array}{l}\text { Coordination des } \\
\text { opérations } \\
\text { logistiques }\end{array}$ & $\begin{array}{l}\text { Cultura : "Si la commande web en magasin est passée avant 16h, elle est livrée le lendemain en magasin avant 10h ; elle nécessite encore un } \\
\text { paiement en caisse et la création d'un compte client sur le site web. " } \\
\text { Aldo : "Parfois le magasin qui reçoit la commande doit la décliner car la chaussure est en fait abimée. Parfois, ils le font pour de mauvaises } \\
\text { raisons (fin de la journée, je suis fatigué). Le système doit donc trouver le magasin le plus proche qui dispose du produit et pourrait l'expédier. Si } \\
\text { tel est le cas, on perd en moyenne } 12 \text { heures [...] Avant, l'achat de produit pour l'e-commerce était distribué dans les différents magasins, } \\
\text { maintenant il est pour une grande partie stocké dans nos entrepôts, ce qui se traduit, pour les magasins, par une baisse d'inventaire et donc de } \\
\text { l'offre disponible. [...] Dans le cas de l'e-réservation, le magasin accomplit une tâche qui ne va peut-être pas aboutir à une transaction (si le client } \\
\text { ne se présente pas) et qui risquerait de priver un client en magasin d'un produit qu'il voudrait acheter. » } \\
\text { Spartoo : «On aimerait pouvoir exploiter davantage les synergies liées à notre important stock web afin de pouvoir faire tourner les produits en } \\
\text { magasin plus souvent et rapidement (à la différence d'un retailer physique qui doit garder en stock toute une saison les produits qu'il a acheté). " } \\
\text { Nicolas : "Nos cavistes même s'ils s'entendent bien, sont quand même en concurrence pour pouvoir faire du store-to-store (livraisons inter- } \\
\text { magasins). " }\end{array}$ \\
\hline
\end{tabular}

Tableau 7 - Les ressources relationnelles 


\subsection{Les ressources d'expertise}

Les ressources d'expertise renvoient à la capacité des entreprises à développer les compétences humaines et à intégrer des équipes munies des connaissances et expériences techniques indispensables. Deux catégories de ressource d'expertise sont identifiées dans le verbatim : la formation et les connaissances individuelles (Tableau 8). Les actions de formation et les connaissances individuelles constituent des ressources facilitant l'intégration des canaux en réduisant les phénomènes de résistance de la part de certaines catégories de salariés.

Les mesures de formation constituent une condition nécessaire du succès des stratégies d'intégration. Elles sont fréquemment évoquées. Le personnel formé devient lui-même une ressource incontournable, source d'avantage concurrentiel. Toutes les enseignes soulignent la nécessité de former en interne. Dans un premier temps, cette formation est générale et porte sur l'évolution du commerce et elle s'adresse à tous. La formation s'adapte ensuite en fonction des métiers. Les vendeurs font l'objet d'une attention particulière : modules de e-learning (Cultura, Nicolas), démonstration en magasin (Cultura) et coaching personnalisé pour les plus résistants (Cultura).

Les connaissances individuelles constituent une ressource précieuse pour le succès de l'intégration des canaux. L'affinité avec le digital est ainsi mentionnée comme facteur favorisant l'adaptation au changement par deux distributeurs (SAQ et Spartoo) alors que le manque d'affinité joue comme un frein pour un autre distributeur (Cultura). Certains distributeurs mentionnent un effet lié à l'âge des vendeurs qui peut jouer favorablement chez Aldo («Nos vendeurs, tout comme nos clients, sont relativement jeunes et ne sont donc pas réfractaires à la technologie. ») ou défavorablement comme chez Nicolas ("c'est aussi une question de génération. Une fois que l'on a réussi à expliquer que c'est complémentaire [Internet et magasin], ça a été plutôt bien accueilli. ») Les phénomènes de résistance ont été plus rares chez la SAQ et Spartoo. Dans tous les cas, les actions d'information et de formation, sont nécessaires pour permettre aux collaborateurs d'acquérir les connaissances nécessaires à l'intégration des canaux (Cultura, SAQ, Nicolas, Spartoo). 


\begin{tabular}{|c|c|}
\hline Facettes & Verbatim \\
\hline $\begin{array}{l}\text { Mesures de } \\
\text { formation }\end{array}$ & $\begin{array}{l}\text { Cultura : "Le premier changement à piloter aujourd'hui c'est expliquer aux collaborateurs ce qu'est le commerce aujourd'hui et comment } \\
\text { fonctionnent les clients. [...] Cette démarche de commerce connecté nécessite une phase d'information, de formation et d'appropriation des } \\
\text { conseillers de vente car elle entraine une évolution de la manière dont ils réalisent leur métier. Cela s'inscrit dans la démarche plus globale de } \\
\text { la transformation digitale de l'entreprise. [...] Le vendeur connecté chez Cultura s'est approprié, dans un premier temps, le terminal mobile } \\
\text { connecté. Dans un second temps, les conseillers de vente en magasin ont découvert l'intérêt d'un point de vue pratique de la commande web en } \\
\text { magasin. Ces changements sont accompagnés par des formations / démonstrations en magasin et reposent également sur des modules d'e- } \\
\text { learning (le client 2.0, le commerce digital...). } \\
\text { Aldo : «Le développement vers l'omnicanal nécessite d'acculturer toute l'entreprise à cette démarche. [...] Il nous a fallu communiquer et former } \\
\text { les magasins sur cet atout supplémentaire pour servir et satisfaire leurs clients en magasin. Un client, qu'il soit en magasin ou sur Internet est } \\
\text { un client d'Aldo et pourra (re)venir acheter dans le magasin ou en ligne s'il est satisfait. » } \\
\text { Nicolas : «le siège a dû convaincre les cavistes que le site n'était pas une concurrence en tant que tel mais plutôt un outil pour travailler sur } \\
\text { l'omnicanal et l'interaction entre le réseau physique et les outils digitaux [...] Il a fallu l'expliquer [...] On a un site e-learning en interne où il } \\
\text { y a des petits quizz pour améliorer leurs connaissances, [...] Ils se forment eux même entre deux ventes. [...] Formations généralistes possibles, } \\
\text { pour le moment. [...] Formations e-consommateurs, etc., vont être déployées quand nous auront mis en place le wallet, la e-livraison » }\end{array}$ \\
\hline $\begin{array}{l}\text { Connaissances } \\
\text { individuelles }\end{array}$ & $\begin{array}{l}\text { Cultura : "Certaines équipes en magasin, suivant leur affinité avec le digital, ont encore du mal à utiliser leur assistant numérique et ont toujours } \\
\text { recours au poste informatique présent au point d'accueil dans le rayon." } \\
\text { Aldo : "Il y a encore certaines divisions de l'entreprise qui ne sont pas familières avec les spécificités des activités e-commerce et du client e- } \\
\text { commerce par rapport au client en magasin." } \\
\text { Spartoo : "Pour le lancement de ses magasins, Spartoo s'est basé sur son expérience web. [...] Ils [les vendeurs] sont sensibilisés sur le fait que } \\
\text { ces ventes contribuent au business global de l'entreprise." } \\
\text { SAQ : "Les employés sont très ouverts à la digitalisation du point de vente et en sont même demandeurs. [...] Face au développement des } \\
\text { commandes en ligne qui risquent de faire diminuer les ventes en boutique, les vendeurs comprennent bien l'intérêt du click-\&-collect et sont } \\
\text { fortement motivés pour engager la discussion avec le client qui vient récupérer son colis afin de lui proposer une dégustation, lui faire découvrir } \\
\text { les nouveautés et générer du CA additionnel. Il est préconisé que le vendeur ouvre et vérifie la commande avec le client dans la boutique.» }\end{array}$ \\
\hline
\end{tabular}

Tableau 8 - Les ressources d'expertise 


\section{Discussion}

L'étude empirique confirme la nécessité, pour les distributeurs engagés dans une stratégie d'intégration des canaux, de disposer de ressources variées. La conduite de la présente réflexion sur les ressources, effectuée selon les axes de la grille proposée par Duong et Paché (2015) développée dans le contexte de la logistique, se révèle une méthode pertinente. En effet, l'étude exploratoire menée auprès de six distributeurs, français et canadiens, appartenant à trois secteurs d'activité différents, permet de vérifier que les cinq catégories de ressources identifiées par Duong et Paché (2015) pour la logistique peuvent être transposées à l'intégration des canaux de distribution.

Ainsi, d'un point de vue théorique, cet article a fait émerger et structuré une réflexion autour des ressources nécessaires au déploiement d'une stratégie omnicanal, en s'appuyant sur le cadre théorique de la RBV. Il ressort des résultats que les ressources technologiques, organisationnelles, physiques, relationnelles et d'expertise peuvent représenter une source d'avantage concurrentiel durable pour un distributeur, notamment en situation de forte concurrence comme souligné par Duong et Paché (2015). D'un point de vue managérial, cet article souligne la diversité des ressources qui sous-tend les stratégies d'intégration des canaux et fournit aux équipes dirigeantes un outil permettant d'établir un diagnostic des ressources.

Pour chacune des cinq ressources, des contributions théoriques et managériales peuvent être soulignées. Dans un souci de synthèse, au sein des cinq catégories de ressources, l'accent est mis sur celles qui ressortent comme des ressources clés. Les contributions théoriques et managériales s'accompagnent de préconisations à l'égard des entreprises du secteur de la distribution d'ores et déjà engagées ou sur le point de s'engager dans une stratégie d'intégration des canaux de distribution. Le tableau ci-dessous (Tableau 9) synthétise les principales contributions théoriques et managériales pour chaque ressource, qui sont ensuite détaillées dans les paragraphes ci-dessous.

\begin{tabular}{|c|c|c|}
\hline Ressource & Contributions théoriques & Contributions managériales \\
\hline $\begin{array}{l}\text { Ressources } \\
\text { technologiques }\end{array}$ & $\begin{array}{l}\text { L'intégration des SI est la } \\
\text { ressource clé et dépend de l'ADN } \\
\text { du distributeur (click \& mortar vs. } \\
\text { pure player). }\end{array}$ & $\begin{array}{l}\text { Il est nécessaire de disposer d'un } \\
\text { SI adapté au partage de données } \\
\text { issues des multiples canaux. }\end{array}$ \\
\hline $\begin{array}{l}\text { Ressources } \\
\text { organisationnelles }\end{array}$ & $\begin{array}{l}\text { Quatre types de ressources sont } \\
\text { nécessaires : la culture } \\
\text { d'entreprise, l'implication des } \\
\text { équipes dirigeantes, la } \\
\text { coordination intra- et inter- } \\
\text { organisationnelle et les processus } \\
\text { commerciaux. }\end{array}$ & $\begin{array}{l}\text { Il est recommandé de mettre en } \\
\text { place une coopération entre les } \\
\text { canaux pour casser l'idée de leur } \\
\text { mise en concurrence. }\end{array}$ \\
\hline $\begin{array}{l}\text { Ressources } \\
\text { physiques }\end{array}$ & $\begin{array}{l}\text { Deux types de ressources sont } \\
\text { essentielles : les surfaces } \\
\text { d'entreposage et le nombre de } \\
\text { points de vente. L'aménagement } \\
\text { du point de vente apparait moins } \\
\text { déterminant. }\end{array}$ & $\begin{array}{l}\text { Le nombre de points de vente et } \\
\text { leur localisation représentent des } \\
\text { enjeux cruciaux pour un } \\
\text { détaillant souhaitant intégrer les } \\
\text { canaux physiques et digitaux. }\end{array}$ \\
\hline
\end{tabular}




\begin{tabular}{|l|l|l|}
\hline $\begin{array}{l}\text { Ressources } \\
\text { relationnelles }\end{array}$ & $\begin{array}{l}\text { La capacité à instaurer une } \\
\text { relation de confiance entre les } \\
\text { canaux et à coordonner } \\
\text { intelligemment les opérations } \\
\text { logistiques entre ces derniers } \\
\text { constituent des ressources clés. }\end{array}$ & $\begin{array}{l}\text { La coordination optimisée des } \\
\text { opérations logistiques représente } \\
\text { un enjeu important. En ce qui } \\
\text { concerne la confiance entre } \\
\text { canaux, les résultats mettent au } \\
\text { jour une relative méfiance entre } \\
\text { ces derniers. }\end{array}$ \\
\hline $\begin{array}{l}\text { Ressources } \\
\text { d'expertise }\end{array}$ & $\begin{array}{l}\text { Le management des RH joue un } \\
\text { rôle essentiel lors des stratégies } \\
\text { d'intégration des canaux afin } \\
\text { d'obtenir l'adhésion du personnel } \\
\text { en magasin et éviter les risques de } \\
\text { résistance face à la technologie. }\end{array}$ & $\begin{array}{l}\text { Un plan d'accompagnement du } \\
\text { changement doit être déployé } \\
\text { (phase d'information et actions } \\
\text { de formation adaptées à chaque } \\
\text { métier impacté par l'intégration } \\
\text { des canaux). }\end{array}$ \\
\hline
\end{tabular}

Tableau 9 - Synthétise des contributions théoriques et managériales par ressource

\subsection{Les ressources technologiques}

Parmi les ressources technologiques, l'intégration des SI ressort comme étant la ressource clé, bien que n'étant pas la seule ressource nécessaire. En effet, les six distributeurs participant à cette étude considèrent l'intégration des SI comme une des ressources centrales sur la base de laquelle il est possible d'acquérir des compétences potentiellement source d'un avantage concurrentiel. Ces compétences peuvent prendre la forme d'une meilleure connaissance des clients cross-canaux qui permet, par exemple d'adapter l'assortiment des magasins en s'appuyant sur les achats en ligne de la zone de chalandise. L'intégration des SI permet aussi de garantir une grande fiabilité des informations en temps réel telles que la disponibilité réelle des marchandises en prenant en compte à la fois les stocks des entrepôts centralisés, mais aussi les stocks en magasins. L'intégration des SI donne, en outre, «des armes » nouvelles au vendeur en magasin qui peut, depuis une tablette ou un smartphone accéder en l'intégralité de l'historique du client qui se rend en boutique et lui proposer des produits additionnels (si le client vient retirer en magasin, un achat ou une réservation réalisés en ligne) ou des produits de substitution si le produit à retirer ne lui convient plus, réduisant ainsi le manque à gagner.

Bien que le principe d'une intégration des SI soit reconnu comme un levier incontournable de l'intégration, pour les six enseignes, sa mise en œuvre est très variable. Cette démarche d'intégration semble être plus facile pour les distributeurs ayant un ADN de pure player que pour ceux issus du commerce traditionnel. Cela ressort à la fois du discours des distributeurs traditionnels devenus click \& mortar, mais également des propos de Spartoo qui a l'ADN d'un pure player. La question de l'intégration du SI est centrale chez les premiers, quel que soit le secteur comme souligné par Lewis et al. (2014). La seule enseigne à ne pas être confrontée à cette question est Spartoo. L'intégration des SI s'est imposée comme une évidence dès l'ouverture de ses premières boutiques, de par son ADN de pure player.

D'un point de vue managérial, s'il est crucial de s'assurer, a priori, de disposer d'un SI adapté au partage de données issues des multiples canaux, cela ne garantit pas d'acquérir une compétence centrale. La technologie ne se suffit pas à elle-même. Les occasions de collecte de données doivent être clairement identifiées (par exemple, enregistrer un passage en magasin qu'il ait donné lieu ou non à un achat). Des procédures systématiques de collecte de données doivent être déployées afin d'alimenter les bases de données et d'acquérir de la connaissance 
client. JouéClub, choisi comme exemple introductif, illustre la nécessité d'intégrer les SI et les bases de données de l'ensemble du réseau.

Un autre élément clé des ressources technologiques concerne la Technologie Digitale en Magasin. Une réflexion doit être engagée pour savoir dans quelle mesure les différents dispositifs peuvent contribuer à l'acquisition de compétences centrales. Quelle valeur pourraient-ils apporter aux clients et surtout au personnel en magasin ? En quoi, les vendeurs seraient-ils plus performants que les vendeurs d'un concurrent qui ne disposeraient pas de ces dispositifs ? La facilité d'usage et l'utilité pour l'utilisateur doivent guider les choix car ils faciliteront leur adoption. Il ne s'agit pas d'opter pour tel ou tel dispositif par simple mimétisme. Il est aisé de comprendre l'intérêt pour Décathlon de proposer une expérience en réalité augmentée pour que le client se rende mieux compte de l'espace proposé à l'intérieur d'une tente. Cela serait disproportionné dans d'autres situations. Pour certaines marchandises, comme les chaussures, les vêtements ou les lunettes, un essayage assisté par la réalité augmentée pourrait être valorisé par le client qui n'est pas obligé d'essayer tous les modèles et par le responsable d'un magasin qui n'est pas obligé de stocker physiquement tous les modèles dans toutes les tailles. L'article choisi et/ou personnalisé pourrait être commandé en boutique et récupéré en magasin ou livré à domicile. Des applications multimarques sont disponibles sur le marché (Wanna Kicks, pour un essayage virtuel de chaussures, par exemple) ; l'investissement peut donc être limité.

\subsection{Les ressources organisationnelles}

Les résultats ont permis d'identifier quatre types de ressources organisationnelles : la culture d'entreprise, l'implication des équipes dirigeantes, la coordination intra- et interorganisationnelle et les processus commerciaux.

D'un point de vue théorique, les résultats de notre étude exploratoire soulignent l'importance cruciale de la culture d'entreprise en tant que ressource. Un effet de la culture d'entreprise semble émerger : Les distributeurs issus du commerce traditionnel ont tendance à considérer que leur culture « magasin » agit comme un frein à l'intégration des canaux, alors que la culture digitale y serait favorable et faciliterait l'adoption des TDM. Lewis et al. (2014) observaient qu'en fin de processus, des problèmes liés à la culture et à l'engagement du personnel devaient être surmontés. En revanche, les distributeurs que nous avons interrogés n'ont pas témoigné d'un changement de culture, comme cela a pu être relevé dans la littérature (Lewis et al., 2014). Cela ne signifie pas pour autant qu'un tel changement ne soit pas à l'œuvre. Lorsque nous avons réalisé la collecte des données, les enseignes n'étaient pas encore arrivées au terme du processus d'intégration.

Les résultats de l'étude empirique mettent la lumière sur une seconde ressource organisationnelle : l'implication de l'équipe dirigeante. L'intégration des canaux de distribution s'inscrit dans le cadre plus global de la transition digitale de l'entreprise défini par Dudézert (2018) comme «l'exploration et l'exploitation des nouveaux possibles engendrés par les TI, en particulier au niveau organisationnel ». Afin d'aborder cette transition digitale et notamment l'intégration des canaux de distribution, il est nécessaire de se situer à un niveau stratégique car cette intégration s'accompagne nécessairement d'une collaboration inter-fonctionnelle. L'implication des équipes dirigeantes ressort comme une ressource clé de l'intégration des canaux. Sans celle-ci, il est probable que l'intégration ne puisse pas avoir lieu. Les résultats confirment que la décision d'intégrer les canaux relève de la stratégie. Elle est prise au plus haut niveau (entérinée par le Comité de Direction de la SAQ, par exemple) et se traduit par une modification de la structure de certains distributeurs (création, chez Cultura, d'une direction du commerce connecté rattachée directement à la direction générale). 
Les résultats confirment également la coordination intra-organisationnelle comme ressource clé de l'intégration des canaux. La question de la coordination inter-organisationnelle est plus rarement évoquée, mais la forte concurrence perçue en interne entre canaux web et magasins fait que cette coordination tend à se rapprocher d'une coordination inter-organisationnelle, dans le cas particulier de l'intégration des canaux. Fabbe-Costes (2002a) souligne que, face à des SI conçus initialement de manière autonome, la coordination intra-organisationnelle était difficile à atteindre, mais faisable. Selon ce même auteur, il doit être envisagé de passer d'un paradigme transactionnel (fondé sur l'échange d'informations nécessaires à des transactions clairement identifiées, par exemple, passer une commande) à un paradigme relationnel, « car la gestion des relations entre parties prenantes d'une supply chain s'accompagne d'une communication qui va au-delà des seules transactions » (Fabbe-Costes, 2002a, p. 82).

Il est présomptueux de formuler des recommandations permettant de faire évoluer la culture d'entreprise vers une culture compatible avec l'intégration des canaux. En revanche, d'autres ressources organisationnelles peuvent être utilisées comme des leviers qui progressivement feront évoluer cette culture. Par exemple, définir des processus rendant plus systématique l'enregistrement de clients magasin dans la base clients, ou permettant de proposer des offres complémentaires adaptées (en ayant informé les vendeurs de l'utilité de telles pratiques) peut amener peu à peu les vendeurs à s'imprégner d'une culture orientée clients.

Traditionnellement et particulièrement en phase de démarrage d'intégration des canaux, le sentiment qui domine est souvent celui d'une mise en concurrence. Il est donc recommandé de tout mettre en ouvre pour créer un climat de confiance entre canaux. Cet objectif peut être atteint en définissant au préalable les principes d'une forme de coopération entre canaux pour casser l'idée d'une mise en concurrence. Il est, par exemple, important d'engager une réflexion sur la manière de compenser le temps consacré en magasin à la préparation des commandes passées en ligne, ou sur la manière d'attribuer le chiffre d'affaires associé aux commandes web passées en magasin ou celles qui sont récupérées en click-\&-collect ou suite à une e-réservation. Les détaillants souhaitant s'engager vers une stratégie d'intégration des canaux peuvent s'inspirer des pratiques mises en place par Cultura ou Aldo.

\subsection{Les ressources physiques}

Les résultats mettent en évidence deux types de ressources physiques essentielles : les surfaces d'entreposage et le nombre de points de vente. L'aménagement (ou le réaménagement) du point de vente apparait, quant à lui, moins déterminant ; il s'agit plus d'une conséquence de la TDM.

Les magasins en tant que point de vente, de retrait, de retour ou d'entreposage de marchandises et les surfaces d'entreposage centralisées destinés au e-commerce ou au réapprovisionnement des magasins, constituent des ressources qui sont au cœur du dispositif omnicanal. Ces ressources physiques représentent des éléments d'infrastructure nécessaires au fonctionnement de la chaîne logistique, permettant de livrer des produits aux clients quand ils le souhaitent, là où ils le souhaitent et comme ils le souhaitent, comme le préconisent Hübner et al. (2016).

La localisation des surfaces d'entreposage constitue une ressource primordiale pour les six distributeurs étudiés entraînant parfois des relocalisations stratégiques (Aldo). Ces entrepôts peuvent servir parfois au réapprovisionnement des magasins et à la préparation des commandes web (De Serres). De plus, un nombre important de points de vente est présenté comme une ressource clé car, outre leur fonction première de vente, ils permettent d'améliorer la connaissance client (Cultura) et constituent potentiellement des surfaces de stockage additionnelles lorsque la livraison depuis le magasin ou la livraison de magasin à magasin est envisagée (SAQ). Aucune enseigne étudiée n'envisage de réduire le nombre de ses points de vente. L'ouverture de magasins était incontournable pour le pure player Spartoo et il envisage de poursuivre le développement de son réseau physique. 
D'un point de vue managérial, le nombre de points de vente et leur localisation représentent des enjeux cruciaux pour un détaillant souhaitant intégrer les canaux physiques et digitaux. C'est, en effet, l'un des investissements parmi les plus lourds. Si le nombre de magasins est jugé insuffisant, des ouvertures devront être envisagées. Se pose alors la question des modalités possibles : ouverture pure et simple, rachat ou partenariat avec des détaillants existants. L'alliance conclue entre Amazon et Monoprix en mars 2018, pour l'offre alimentaire, constitue un bon exemple d'intégration du physique et du digital : le picking est assuré par les magasins Monoprix et la livraison par Amazon. Amazon peut ainsi intégrer une offre alimentaire dans le programme Prime sans avoir à investir dans une infrastructure ni à négocier avec les grandes marques pour obtenir un référencement, alors que Monoprix peut, cibler de nouveaux clients et enrichir son offre de services.

Un aménagement du point de vente adapté à l'intégration des canaux constitue une ressource essentielle, bien qu'il ne soit pas toujours perçu comme tel par les détaillants considérés dans cette recherche. Si le diagnostic fait ressortir que l'aménagement actuel des magasins n'est pas optimisé pour l'accueil des clients venant récupérer une commande click-\&-collect ou pour développer les commandes web en magasin, de nouveaux concepts de magasins doivent être imaginés en amont. L'intégration des canaux peut même constituer une opportunité propice à l'innovation. Undiz constitue de ce point de vue un cas d'école : des contraintes de surface ont conduit l'enseigne à développer un concept de magasin très innovant et incorporant une forte dose de Technologie Digitale en Magasin, la «Undiz Machine». Sans cela, Undiz aurait dû renoncer à un local très attractif à Toulouse.

\subsection{Les ressources relationnelles}

Sur le plan théorique, les ressources relationnelles font référence à la capacité d'une entreprise à construire des relations étroites avec ses partenaires à travers la collaboration et la confiance en vue de se coordonner, de partager des informations et de satisfaire les demandes de leurs clients. Les résultats exploratoires montrent que la capacité à instaurer une relation de confiance entre les canaux et à coordonner intelligemment les opérations logistiques entre ces derniers constituent effectivement des ressources relationnelles clés. Dans le cadre d'une intégration omnicanal, des canaux qui pouvaient être perçus comme concurrents (magasin - Internet) sont amenés à collaborer. La question de l'échange d'information entre canaux est cependant peu évoquée par les entreprises étudiées.

Ainsi, une coordination optimisée des opérations logistiques peut rééquilibrer la relation entre les canaux et constitue, en ce sens, une ressource. Dans le cadre de la logistique de sortie, la coordination intra-organisationnelle doit permettre un équilibre entre les expéditions depuis les magasins ou depuis les entrepôts centralisés. Les arbitrages chez les distributeurs interrogés se font en prenant en compte le lieu de livraison choisi par le client (domicile ou point de vente), la proximité des stocks (magasin ou entrepôt centralisé), la disponibilité des marchandises, la capacité de traitement des commandes au point de vente. Tchokogué et al. (2008) identifiaient comme barrières entravant l'intégration de l'entreprise, les barrières physiques dues à la distance séparant la localisation géographique des entités d'une entreprise. Cette difficulté semble plus marquée au Canada.

Outre la classique logistique de sortie, les distributeurs sont confrontés à une problématique de logistique des retours. Les consommateurs sont demandeurs de dispositifs simples et pratiques pour retourner les marchandises commandées en ligne, lorsqu'elles ne conviennent pas. Ces questions sont au cœur des préoccupations des distributeurs interrogés par Hübner et al. (2016). On les retrouve chez les distributeurs interrogés dans cette étude. Donner la possibilité de retour en magasin des marchandises commandées en ligne, si elle est souhaitable du point de vue de l'expérience client, peut être l'occasion de frictions, surtout si cela se fait sans contrepartie. 
Chez Cultura, les magasins se retrouvaient parfois devoir gérer les retours des produits achetés en ligne, mais ne bénéficiaient pas des promotions proposées uniquement sur le web.

Si la confiance entre canaux physique et virtuel facilite le déploiement de la stratégie d'intégration, la plupart des distributeurs étudiés de type click-and-mortar (Cultura, Aldo, SAQ, Nicolas) ont connu, à un moment où à un autre, une perte de confiance : la vente en ligne était perçue comme une menace entraînant des phénomènes de résistance. Comme l'ont souligné Lewis et al. (2014), c'est au cours de la phase intermédiaire de l'intégration des canaux que le sentiment de mise en concurrence entre les canaux est le plus exacerbé chez les vendeurs en magasin des enseignes puisant leurs racines dans le commerce physique. De plus, la confiance entre les clients et les vendeurs constitue également une ressource relationnelle pour Nicolas et Cultura. Pour ce dernier, l'acte de vente n'est plus une relation «face-à-face » mais une relation « côte-à-côte ».

D'un point de vue managérial, la coordination optimisée des opérations logistiques représente un enjeu important. L'intégration a pour conséquence la prise en charge par les magasins de tâches logistiques nouvelles comme la préparation des commandes en ligne, le retrait en magasin et/ou la gestion des retours. Sur ce point particulier, le cas d'Aldo est riche d'enseignements. Faire reposer la logistique des commandes en ligne presque exclusivement sur les magasins s'est avéré contreproductif. Il est donc recommandé de définir au préalable un équilibre afin de ne pas donner l'impression aux vendeurs en magasin qu'ils disposent de moins de temps pour servir les clients qui se déplacent en magasin. Un nombre optimal de préparations de commande en magasin pourrait être défini, au-delà duquel il serait possible de refuser de nouvelles préparations. De même, une réflexion doit être menée de manière à réduire le risque de rupture de stock en magasin. Des mécanismes de stock de sécurité et/ou de livraison de magasin à magasin pourraient être mis en place pour éviter les ventes perdues faute de marchandise disponible au point de vente. Enfin, le succès de l'intégration des canaux repose également sur une forme de confiance intra-organisationnelle : la confiance entre canaux. Or, les résultats mettent au jour une relative méfiance entre ces derniers. Les actions à mener se situent essentiellement au niveau de la formation (ce point est développé à propos des ressources d'expertise, ci-dessous).

\subsection{Les ressources d'expertise}

Pour rappel, les ressources d'expertise renvoient à la capacité des entreprises à développer les compétences humaines et à intégrer des équipes munies des connaissances et expertises techniques indispensables. Elles sont donc étroitement liées aux RH. De manière générale, les résultats confirment le rôle essentiel des $\mathrm{RH}$ lors des stratégies d'intégration des canaux de distribution. Les distributeurs ont conscience de la nécessité d'obtenir l'adhésion du personnel en magasin pour mener à bien cette stratégie. L'intégration des canaux de distribution suppose un recours accru aux TI et les résultats confirment le risque de résistance que cela entraine, conformément à la littérature en SI (Davis, 1989) et à celle centrée sur l'intégration des canaux (Spreer et Rauschnabel, 2016). Spreer et Rauschnabel (2016) identifiaient six facteurs de résistance à l'adoption par les vendeurs de tablettes ou de smartphones, parmi lesquels la peur d'être remplacé par la technologie, Les responsables de Cultura y voit, au contraire, un moyen de rééquilibrer la relation entre vendeur et client. Face aux clients « augmentés » qui peuvent accéder en permanence à des informations permettant de positionner une offre donnée par rapport aux concurrents (en ligne ou dans un autre magasin), il semble nécessaire de donner aux vendeurs des moyens leur permettant de rivaliser. Un smartphone leur donne non seulement accès aux conditions pratiqués par la concurrence, mais également au profil du client s'il est enregistré dans la base. La vision des cadres de Cultura nécessite cependant un accompagnement des vendeurs en magasin afin que ces derniers puissent saisir l'intérêt de tels dispositifs et lever une autre barrière au changement identifiée dans la littérature, la crainte de 
ne pas être compétent et de commettre des erreurs devant le client (Spreer et Rauschnabel, 2016).

Les résultats soulignent le rôle essentiel des ressources d'expertise car elles contribuent à l'acquisition de compétences indispensables à l'intégration de canaux, compétences identifiées dans la littérature comme cross-channel HR capabilities (Payne et Frow, 2004 ; Oh et al. 2016). Les distributeurs interrogés font en sorte de favoriser l'acquisition de ces compétences spécifiques durant les trois stades de l'intégration, conformément au constat opéré par Lewis et al. (2014). Dès le premier stade, l'acquisition de ces compétences se fait soit par le recrutement d'un personnel ayant intégré les principes de l'omnicanal, s'il s'agit de créer ex-nihilo un réseau physique comme pour Spartoo, soit par l'accompagnement au changement du personnel en place dès les prémisses du projet, comme cela a été le cas chez Cultura. C'est au cours de la phase intermédiaire que le sentiment de mise en concurrence entre les canaux est le plus exacerbé chez les vendeurs en magasin des enseignes puisant leurs racines dans le commerce physique. Au cours de cette phase, on observe que les enseignes redoublent d'efforts pour consolider l'acquisition des compétences. C'est à ce moment qu'est inculquée l'idée qu'un client, quel que soit son canal de prédilection, est un client de l'enseigne et qu'il contribue à la performance globale de l'entreprise, comme cela est fait chez Aldo. Enfin, c'est durant le stade avancé de mise en œuvre, qu'une nouvelle culture est susceptible d'émerger sur la base des compétences acquises. Là aussi, il semble que ce soit le cas dans les magasins Aldo.

D’un point de vue managérial, étant donné les phénomènes de résistance observés dans l'étude empirique, il est recommandé de prévoir un plan d'accompagnement du changement. Celui-ci doit inclure une phase d'information, au plus tôt, puis des actions de formation adaptées à chaque métier impacté par l'intégration des canaux. Pour des aspects généraux, des modules de formation en ligne peuvent suffire, mais pour une meilleure maîtrise des outils (bornes, tablettes, miroir connecté, applications de réalité augmentée, etc.), une formation avec mise en situation est probablement préférable. Elle facilitera la familiarisation, soulignera l'utilité des dispositifs déployés. De plus, les retours des futurs utilisateurs permettront de corriger d'éventuelles imperfections opérationnelles. Il peut être judicieux, dans un premier temps, de jouer la carte du volontariat et de s'appuyer sur les collaborateurs les plus réceptifs à la technologie. Venkatesh et al. (2003) ont, en effet, montré que l'attitude des salariés à l'égard de la technologie dépend pour partie du fait que l'adoption leur est imposée ou se fait sur la base du volontariat. Les modèles de diffusion des innovations, comme celui proposé par Rogers (1995), montrent que certains individus ont besoin de voir la nouvelle technologie utilisée par d'autres (les innovateurs au sens de Rogers) pour l'adopter eux-mêmes. Les vendeurs-innovateurs pourraient ainsi devenir des ambassadeurs et former leurs collègues.

\section{Conclusion}

En se basant sur le cadre de la théorie des ressources (RBV), cet article a permis de faire émerger et de structurer une réflexion autour des ressources nécessaires au déploiement d'une stratégie omnicanal. Cette réflexion est réalisée selon la classification des ressources logistiques de Duong et Paché (2015) qui est, dans le présent article, utilisée comme grille de lecture et est adaptée à l'intégration des canaux. Différents champs relatifs aux systèmes d'information, à la logistique \& la supply chain et aux ressources humaines ont été retenus. Une étude qualitative exploratoire reposant sur six cas a permis de mettre au jour les ressources déployées dans leurs stratégies d'intégration par six distributeurs français et canadiens dans trois secteurs. Cinq ressources sont identifiées: des ressources technologiques, organisationnelles, physiques, relationnelles et d'expertise. Cette recherche confronte, pour la première fois, la grille de Duong et Paché (2015) à un terrain empirique et élargit son contexte d'application au-delà de son contexte d'origine, la logistique. 
En ce qui concerne les implications managériales, des recommandations peuvent être formulées à destination des dirigeants. En premier lieu, il est préconisé de réaliser un diagnostic. Les distributeurs souhaitant s'engager dans une stratégie d'intégration des canaux doivent, au préalable, dresser un état des lieux des ressources dont ils disposent en interne. Pour mener à bien cette tâche, il est recommandé d'utiliser la grille de lecture de Duong et Paché (2015), telle qu'elle a été adaptée dans cet article, au contexte de l'intégration des canaux. Cet état des lieux permettra de repérer les ressources acquises ou en cours d'acquisition et celles faisant défaut. Il servira ensuite de base à un plan d'actions. Dans un second temps, les équipes dirigeantes devront planifier des actions concrètes permettant l'acquisition ou la consolidation de ressources. Des exemples d'actions à engager ont été proposés pour chaque ressource dans la partie discussion.

Quelques limites sont à souligner. Elles pourraient guider de futures recherches. Tout d'abord, l'étude empirique n'intègre qu'un seul distributeur à l'ADN pure player. Or, il semble que l'intégration des canaux soit plus aisée pour un pure player (SI, RH, etc.). De plus, cinq distributeurs sur six ont opté pour le statut de succursales. La démarche d'intégration des canaux serait-elle identique dans un réseau de franchisés ? Il est donc souhaitable de poursuivre les recherches en se focalisant sur le statut des distributeurs (succursale vs franchise) et la trajectoire suivie (du magasin vers l'omnicanal et vice-versa) afin de tester de manière plus systématique un éventuel effet de ces deux variables. Ensuite, l'interdépendance et la complémentarité des cinq ressources dans le succès de l'intégration des canaux n'est pas examinée. Par ailleurs, l'étude empirique se focalise sur six cas appartenant à trois secteurs. Elle est donc par nature exploratoire. A des fins de validité externe des résultats, il serait donc intéressant d'élargir le terrain d'étude à d'autres entreprise et à d'autres secteurs. Enfin, dans une prochaine recherche, il serait pertinent d'interroger des distributeurs ayant achevé l'intégration des canaux et leur migration vers l'omnicanal afin d'établir un lien entre les ressources et l'acquisition d'un avantage compétitif.

\section{Références}

Adivar, B.; Hüseyinoğlu, I. Ö. Y.; Christopher, M. (2019), “A Quantitative Performance Management Framework for Assessing Omnichannel Retail Supply Chains", Journal of Retailing and Consumer Services, vol. 48, p. 257-269.

Akturk, M. S.; Ketzenberg, M.; Heim, G. R. (2018), "Assessing Impacts of Introducing Shipto-store Service on Sales and Returns in Omnichannel Retailing: A Data Analytics Study", Journal of Operations Management, vol. 61, p. 15-45.

Amit, R.; Schoemaker, P. J. (1993), "Strategic Assets and Organizational Rent", Strategic management journal, vol. $14, \mathrm{n}^{\circ} 1$, p. 33-46.

Arrègle J-L. (1995), «Le savoir et l'approche resource-based: une ressource et une compétence », Revue française de gestion, septembre-octobre, p. 84-94.

Aubrey, C.; Judge, D. (2012), "Re-imagine Retail: Why Store Innovation is Key to a Brand's Browth in the "new normal", Cigitally-connected and Transparent World?", Journal of Brand Strategy, vol. 1, n¹, p. 31-39.

Barney, J. (1991), "Firm Resources and Sustained Competitive Advantage", Journal of Management, vol. 17, $\mathrm{n}^{\circ}$ 1, p. 99-120.

Beck, N.; Rygl, D. (2015), "Categorization of Multiple Channel Retailing in Multi-, Cross-, and Omni-Channel Retailing for Retailers and Retailing", Journal of Retailing and Consumer Services, vol. 27, p. 170-178. 
Bendoly, E.; Blocher, J.D.; Bretthauer, K.M.; Krishnan, S.; Venkataramanan, M.A. (2005) "Online / in-Store Integration and Customer Retention", Journal of Service Research, vol. 7, $\mathrm{n}^{\circ}$ 4, p. 313-327.

Bensaou, M.; Venkatraman, N. (1995) "Configurations of Interorganizational Relationships: A Comparison between US and Japanese Automakers", Management science, vol. 41, $\mathrm{n}^{\circ}$ 9, $\mathrm{p}$. 1471-1492.

Bidan, M. (2004) «Fédération et intégration des applications du système d'information de Gestion », Systèmes d'Information et Management, vol. 9, nº 2, p. 5.

Bidan, M.; Rowe, F.; Truex, D. (2012), “An Empirical Study of IS Architectures in French SMEs: Integration Approaches", European Journal of Information Systems, vol. 21, $\mathrm{n}^{\circ}$ 3, $\mathrm{p}$. 287-302.

Cao L. (2014), "Business Model Transformation in Moving to a Cross-channel Retail Strategy: a Case Study", International Journal of Electronic Commerce, vol. 18, n 4, p. 69-95.

Cao, L.; Li, L. (2015), The Impact of Cross-channel Integration on Retailers' Sales Growth, Journal of Retailing, vol. 91, $\mathrm{n}^{\circ}$ 2, p. 198-216.

Cappiello, C.; Francalanci, C.; Pernici, B. (2003), "Time-related Factors of Data Quality in Multichannel Information Systems, Journal of Management Information Systems, vol. $20 \mathrm{n}^{\circ} 3$, p. 71-92.

Chalmeta, R.; Campos, Ch.; Grangel, R. (2001), "References for Architecture for Enterprise Integration", The Journal of Systems and Software, vol. 57, n 3, p. 175-191.

Davis, F.D (1989), "Perceived Usefulness, Perceived Ease of Use, and User Acceptance of Information Technology", MIS Quarterly, vol. 13 n³, p. 319-340.

De Corbière; F., Rowe, F.; Wolff, F. C. (2012), «De l'intégration interne du système d'information à l'intégration du système d'information de la chaîne logistique », Systèmes d'information \& management, vol. $17, \mathrm{n}^{\circ} 1, \mathrm{p} .81-111$.

Deleersnyder, B.; Geyskens, I.; Gielens, K.; Dekimpe, M. G. (2002), "How Cannibalistic is the Internet Channel? A Study of the Newspaper Industry in the United Kingdom and the Netherlands", International Journal of Research in Marketing, vol. 19, n 4, p. 337-348.

Dudézert A. (2018), «La transformation digital des entreprises », La Découverte.

Duong, H. T. ; Paché, G. (2015), «Théorie des ressources appliquée à la logistique : Une identification de cinq dimensions clés, Logistique \& Management, vol. 23, $n^{\circ} 2$, p. 55-72.

Emrich O.; Paul M.; Rudolph T. (2015), "Shopping Benefits of Multichannel Assortment Integration and Moderating Role of Retailer Type", Journal of Retailing, vol. 91, $\mathrm{n}^{\circ}$ 2, p. 326342 .

Fabbe-Costes N, (2002b), "Évaluer la création de valeur du Supply Chain Management", Logistique et Management, vol. 10, n 1, p. 29-36.

Fabbe-Costes, N. (2002a), «Le pilotage des Supply Chains : Un Défi pour les Systèmes d'Information et de Communication Logistiques, Gestion 2000, vol. 1, p. 75-92.

Fabbe-Costes, N.; Jahre, M. (2008), "Supply Chain Integration and Performance: A Review of the Evidence, The International Journal of Logistics Management, vol. 19, $\mathrm{n}^{\circ}$ 2, p. 130-154.

Falk, T.; Schepers, J.; Hammerschmidt, M.; Bauer, H. H. (2007), "Identifying Cross-channel Dissynergies for Multichannel Service Providers", Journal of Service Research, vol. 10, $\mathrm{n}^{\circ} 2$, p. 143-160. 
Gallino, S.; Moreno, A. (2014), "Integration of Online and Offline Channels in Retail: The Impact of Sharing Reliable Inventory Availability Information, Management Science, vol. 60 $n^{\circ}$ 6, p. 1434-1451.

Goldstein, S.M.; Johnston, R.; Duffy, J.A.; Rao, J. (2002), “The Service Concept: The Missing Link in Service Design Research?", Journal of Operations Management, vol. 20, $\mathrm{n}^{\circ}$ 2, p. 121134.

Gorkhali, A.; Xu, L.D. (2019), "Enterprise Architecture, Enterprise Information Systems and Enterprise Integration: A Review Based on Systems Theory Perspective", Journal of Industrial Integration and Management; vol. 04, $\mathrm{n}^{\circ}$. 2, p. 103-126.

Hammer, M. (2001), “The Superefficient Company”, Harvard Business Review, vol. 79, p. 8291.

Herhausen, D.; Binder, J.; Schoegel, M.; Herrmann, A. (2015), "Integrating Bricks with Clicks: Retailer-level and Channel-level Outcomes of Online-offline Channel Integration”, Journal of retailing, vol. $91, \mathrm{n}^{\circ} 2$, p. 309-325.

Hlady-Rispal M. (2002), «La méthode des cas : application à la recherche en gestion », De Boeck Université.

Hübner A.; Holzpfel A; Kuhn H. (2016), "Distribution Systems in Omni-channel Retailing, Business Research, vol. 9, p. 255-296.

Ishfaq, R.; Defee, C. C.; Gibson, B. J.; Raja, U. (2016), "Realignment of the Physical Distribution Process in Omni-channel Fulfillment", International Journal of Physical Distribution \& Logistics Management, vol. 46, n 6/7, p. 543-561.

Jeanpert, S.; Paché, G. (2016), "Successful Multi-channel Strategy: Mixing Marketing and Logistical Issues", Journal of Business Strategy, vol. 37, n 2, p.12-19.

Larke, R.; Kilgour, M.; O'Connor, H. (2018), "Build Touchpoints and They will come: Transitioning to Omnichannel Retailing", International Journal of Physical Distribution \& Logistics Management, Vol. 48 n . 4, p. 465-483.

Le Moigne, J.-L. (2012), Les épistémologies constructivistes, PUF, Paris, 127 p.

Lewis J.; Whysall P.; Foster C. (2014), "Drivers and Technology-related Obstacles in Moving to Multichannel Retailing", International Journal of Electronic Commerce, vol. 18, n 4, p. 4367.

Luo, J.; Fan, M.; Zangh, H. (2016), "Information Technology, Cross-Channel Capabilities, and Managerial Actions: Evidence from the Apparel Industry", Journal of the Association for Information Systems, vol. $17 \mathrm{n}^{\circ}$ 5, p. 308-327.

Maiga A.S.; Nilsson A.; Ax C. (2015), "Relationships between Internal and External Information Systems Integration, Cost and Quality Performance, and Firm Profitability", International Journal Production Economics, vol. 169, p. 422-434.

Markus, M.L. (2000), "Paradigm Shifts: E-business and Business/systems Integration", Communications of the Association for Information Systems, vol. 4, $\mathrm{n}^{\circ}$ 10, p. 1-44.

Mollenkopf, D.A.; Rabinovich, E.; Laseter, T.M.; Boyer, K.K. (2007), "Managing Internet Product Returns: a Focus on Effective Service Operations", Decision Sciences, vol. 38, n 2, p. 215-250. 
Oh, L.-B.; Teo, H.-H.; Sambamurthy, V. (2012), "The Effects of Retail Channel Integration through the Use of Information Technologies on Firm Performance", Journal of Operations Management, vol. 30, p. 368-381.

Payne, A.; Frow, P. (2004), "The Role of Multichannel Integration in Customer Relationship Management”. Industrial Marketing Management, vol. 33 n 6, p.527-538.

Penrose, E. T. (1959), “The Theory of the Growth of the Firm”, New York: John Wiley.

Piotrowicz W.; Cuthbertson R. (2014), "Introduction to the Special Issue Information Techenology in Retail: Toward Omnichannel Retailing", International Journal of Electronic Commerce, vol. 18, $\mathrm{n}^{\circ} 4$, p. 5-15.

Prahalad, C. K.; Hamel, G. (1990), «Les grands groupes ne connaissent pas leur métier ». Havard L'Expansion, hiver, 1991.

Reix, R.; Fallery, B.; Kalika, M.; Rowe, F. (2016), « Systèmes d'Information et Management

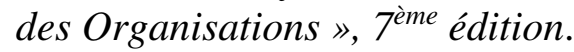

Rigby, D (2011), “The Future of Shopping," Harvard Business Review, vol. 89, n 12, p. 6576.

Rogers, E. (1995), Diffusion of innovations, The Free Press, New York

Saghiri, S.; Wilding, R.; Mena, C.; Bourlakis, M. (2017), "Toward a Three-dimensional Framework for Omni-channel”, Journal of Business Research, vol. 77, p. 53-67.

Seddon, P.; Calvert, C.; Yang, S. (2010), "A Multi-Project Model of Key Factors Affecting Organizational Benefits from Enterprise Systems", MIS Quarterly, vol. 34, n 2, p. 305-28.

Sirmon, D.G.; Hitt, M.A.; Ireland, R.D., (2007), "Managing Firm Resources in Dynamic Environments to Create Value: Looking Inside the Black Box", Academy of Management Review, vol. 32, $\mathrm{n}^{\circ}$ 1, p. 273-292.

Spreer, P.; Rauschnabel, P.A. (2016), "Selling with Technology: Understanding the Resistance to Mobile Sales Assistant Use in Retailing", The Journal of Personal Selling \& Sales Management, vol. 36, $\mathrm{n}^{\circ}$ 3, p. 240-263.

Tate, M.; Hope, B.; and Coker, B. (2005), "The Buywell Way: Seven Essential Practices of a Highly Successful Multi-channel e-Tailer”, Australia Journal of Information Systems, vol. 12, $\mathrm{n}^{\circ} 2$, p. 147-163.

Tchokogué, A.; Perez, M.; Hien, N. (2008), « Mécanismes et niveau d'intégration organisationnelle de l'entreprise : une évaluation empirique avant et après la mise en place d'un système ERP », Systèmes d'information \& management, vol. 13, n 2, p. 61-96.

Tixier, D., Mathe, H., Colin, J. (1996). «La logistique d'entreprise : vers un management plus compétitif », Dunod, Paris.

Truman, G.E. (2000), "Integration in Electronic Exchange Environments", Journal of Management Information Systems, vol. 17, $\mathrm{n}^{\circ}$ 1, p. 209-44.

Van Baal, S. (2014), "Should Retailers Harmonize Marketing Variables across their Distribution Channels? An Investigation of Cross-channel Effects in Multi-channel Retailing", Journal of Retailing and Consumer Services, vol. 21, p. 1038-1046.

Venkateshn, V.; Moris, M.G.; Davis, G.B.; Davis, F.D. (2003), "User acceptance of information technology: toward a unified view”, MIS Quarterly, vol. 27, n 3, p. 423-478. 
Verhoef P.C.; Kannan P.K.; Jeffrey I.J. (2015), "From Multi-channel to Omni-channel Retailing. Introduction to the Special Issue on Multi-channel Retailing", Journal of Retailing, vol. $91, \mathrm{n}^{\circ} 2$, p.174-181.

Vickery, S.K.; Jayaram, J.; Droge, C.; Calantone, R. (2003), "The effects of an Integrative Supply Chain Strategy on Customer Service and Financial Performance: An Analysis of Direct versus Indirect Relationships, Journal of Operations Management, vol. 21, n 5, p. 523-539.

Wernerfelt, B. (1984), “A Resource-based View of the Firm”, Strategic management journal, vol. 5, $\mathrm{n}^{\circ}$ 2, p. 171-180.

Xu, J.; Forman, C.; Kim, J. B.; Van Ittersum, K. (2014), “News Media Channels: Complements or Substitutes? Evidence from Mobile Phone Usage, Journal of Marketing, vol. 78, n 4, p. 97 112.

Yin, C.; Zhang, M.; Zhang, Y.; Wu W. (2019), "Business service network node optimization and resource integration based on the construction of logistics information systems", Information Systems and E-Business Management, (In press).

Yin, R. (1990), "Case study research: design and methods", Berverly Hills, CA, Sage Publications.

Zhang, J.; Farris, P.W.; Irvin, J.W.; Kushwaha, T.; Steenburgh, T.J.; Weitz, B.A. (2010), "Crafting Integrated Multichannel Retailing Strategies", Journal of Interactive Marketing; vol. $24, \mathrm{n}^{\circ} 2$, p. $168-180$. 